\title{
Les dessus et les dessous du lait. Sociologie et politique du lait et de ses dérivés en Mongolie
}

The 'upper' and 'lower' parts of milk. Sociology and politics of milk and its derivatives in Mongolia

\section{Sandrine Ruhlmann et Linda Gardelle}

\section{OpenEdition}

Journals

Édition électronique

URL : https://journals.openedition.org/emscat/1974

DOI : 10.4000/emscat.1974

ISSN : 2101-0013

Éditeur

Centre d'Etudes Mongoles \& Sibériennes / École Pratique des Hautes Études

\section{Référence électronique}

Sandrine Ruhlmann et Linda Gardelle, « Les dessus et les dessous du lait. Sociologie et politique du lait et de ses dérivés en Mongolie », Études mongoles et sibériennes, centrasiatiques et tibétaines [En ligne], 43-44 | 2013, mis en ligne le 20 septembre 2013, consulté le 13 juillet 2021. URL : http:// journals.openedition.org/emscat/1974; DOI : https://doi.org/10.4000/emscat.1974 


\title{
Les dessus et les dessous du lait. Sociologie et politique du lait et de ses dérivés en Mongolie
}

The 'upper' and 'lower' parts of milk. Sociology and politics of milk and its

derivatives in Mongolia

\author{
Sandrine Ruhlmann et Linda Gardelle
}

1 Les éleveurs nomades mongols tirent leur subsistance de l'élevage extensif de plusieurs espèces animales. Cet article, consacré à l'étude sociologique et politique de production et de consommation des produits laitiers, repose sur une étude ethnographique de campements d'éleveurs de chevaux, de vaches, de chèvres et de moutons de la région du Hentij, située à l'Est du pays. Les matériaux ont été recueillis de 1999 à 2006, au sein de quatre campements d'éleveurs halh qui nomadisent sur les communes de Mörön (Ruhlmann) et de Žargalthaan (Gardelle) (cf. cartes ci-dessous). Nos enquêtes de terrain ont couvert les quatre saisons de manière à observer un cycle de vie des hommes dans leur environnement. L'été, les campements regroupent quatre ou cinq foyers. L'hiver, les campements se scindent et les éleveurs transhument pour laisser reposer les pâturages estivaux. Ils empruntent alors parfois deux ou trois chameaux à un campement voisin pour transporter leur mobilier en échange d'un service quelconque. Les éleveurs de moutons et de chèvres du campement d'été s'installent dans le Sud de la région, à une centaine de kilomètres, afin de faire paître leurs troupeaux sur des pâturages dont la flore est plus appropriée à leurs besoins. Les autres foyers du campement, quant à eux, s'éloignent d'une trentaine de kilomètres, tout en gardant une distance équivalente par rapport au district (sum, sous-division administrative), où résident les membres sédentaires de leur famille. 
Carte 1. La Mongolie

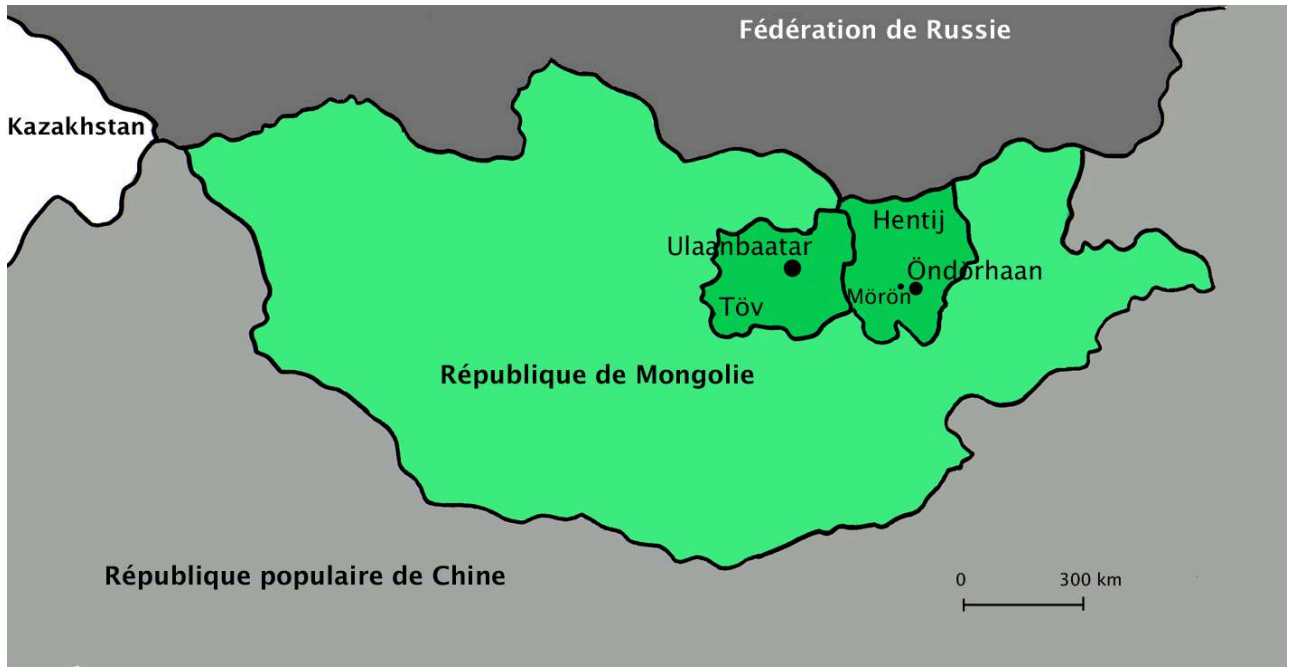

Sandrine Ruhlmann

Carte 2. Le Hentij

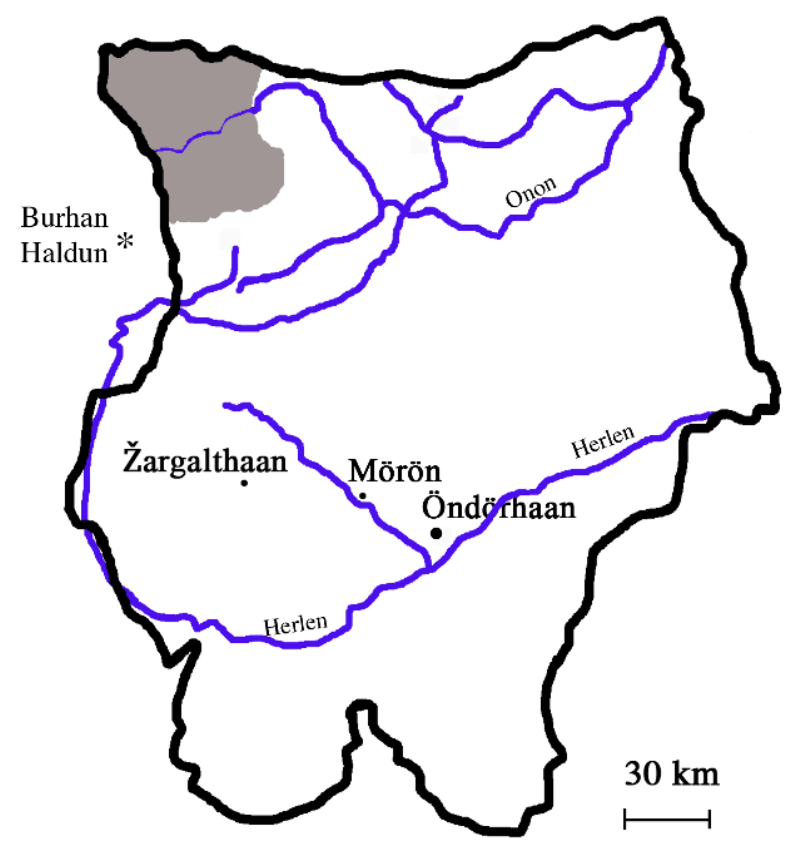

\section{Les monts Hentij}

\section{Sandrine Ruhlmann}

Les techniques de fabrication des produits laitiers révèlent toute une organisation économique et domestique : les femmes traient les femelles des espèces qu'elles élèvent (vache, yak, brebis, chèvre, jument ou chamelle) en fonction du milieu écologique, stockent le lait, le font chauffer et le transforment. Elles détiennent les savoir-faire de fabrication artisanale des produits dérivés du lait, transmis de mère en fille. Nous avons observé et participé à la fabrication de chacun des produits laitiers et, sous une apparente homogénéité, nous constatons des variations de fabrication selon les régions 
et l'espèce animale. Nous avons notamment identifié des spécificités qui visent à modifier légèrement le goût (sucré, salé) et la texture (dure, friable) des produits. La revue Études mongoles a publié en 1975 un article consacré aux activités rurales en République populaire de Mongolie dans les années 1970. Une première partie traite de l'agriculture et de l'élevage mongols: du cadre écologique et des principales caractéristiques des animaux constituant le troupeau, des transformations de l'élevage traditionnel extensif en un élevage de type coopératif sous le régime communiste s'accompagnant de formes de sédentarisation (Accolas \& Deffontaines). Une seconde partie présente une étude de l'économie laitière et de la technologie laitière dans les coopératives (Accolas \& Aubin) ${ }^{1}$. Dans cet article, nous choisissons de présenter une analyse anthropologique de la commensalité des produits laitiers en Mongolie contemporaine, en nous focalisant sur les modalités de distribution, de partage ou d'offrande et de consommation' ${ }^{2}$.

3 Nous montrerons comment les produits laitiers constituent une catégorie d'aliments porteurs de valeurs, de symboles culturels et identitaires en lien avec un passé historique emblématique. Véritable matière sociale, le lait et ses dérivés soutiennent et révèlent un système métaphysique proprement mongol et un mode de relation aux vivants, aux morts, aux esprits-maîtres de la nature et autres entités. Nous partirons des aspects sociologiques, culturels et historiques de la consommation des produits laitiers et nous nous intéresserons également à leurs utilisations rituelles dans des contextes festifs précis. Nous étudierons également le rôle particulier de deux boissons, le lait fermenté et le lait distillé, dans les temps forts de la vie sociale, des éleveurs en particulier et des Mongols en général. Enfin, nous conclurons sur la question de la position du gouvernement à l'égard de l'élevage et des éleveurs au travers des programmes politiques des deux dernières décennies et nous présenterons brièvement le développement et les innovations de l'industrie laitière mongole, pour replacer les pratiques domestiques dans leur contexte économique et politique actuel.

\section{Les modalités d'offrande et de consommation des produits laitiers}

4 Le quotidien des éleveurs, précisément des femmes, est rythmé par les traites et, en été, par la fabrication des produits laitiers et leurs différents modes de conservation (séchage en été, congélation en automne et en hiver) qui leur confèrent une régularité dans la consommation. 
Photo 1. Traite du lait

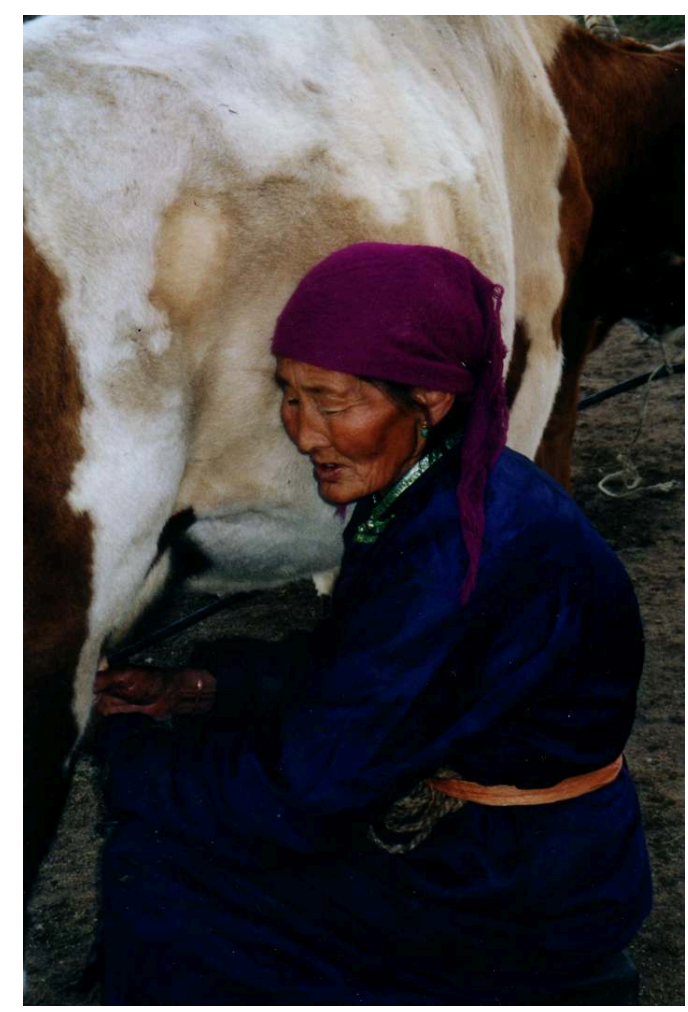

Sandrine Ruhlmann (Hentij, été 2003)

5 Les modes et modalités de consommation ainsi que les manières d'offrir et de manger les produits laitiers montrent que la culture mongole est fondée sur une conception de l'hospitalité typique des éleveurs nomades. Les laitages formés de résidus des produits de base, comme les fromages séchés, sont maigres et considérés comme étant moins nourrissants. Inversement, les produits de base sont gras et nourrissants. De ce fait, les uns sont socialement dépréciés et de cette dévalorisation découle une manière de les présenter, de les offrir et de les consommer qui diffère des autres produits laitiers, qui sont honorifiques.

6 Le lait et les produits dérivés du lait constituent une catégorie d'aliments préférentielle désignée par l'expression "aliments blancs» (cagaan idee) évocatrice de leur couleur. Un passage de l'Histoire secrète des Mongols atteste que le lait symbolisait déjà au XIII ${ }^{\mathrm{e}}$ siècle l'abondance et la fête chez les peuples mongols (Even \& Pop 1994, § 130, 213). Parce qu'ils dérivent du lait, les « aliments blancs » quand ils sont offerts et consommés à l'occasion d'événements festifs renferment alors une symbolique et des caractéristiques appropriées aux exigences rituelles de la fête.

\section{L'offrande matinale : le « dessus » du thé au lait}

7 Tous les matins, les femmes des campements stockent le lait de la traite dans des seaux métalliques au nord-est de la yourte. Quand le thé a infusé, elles ajoutent un demi-litre de lait fraîchement trait pour préparer du « thé avec du lait » (süütej caj). Avec la louche (šanaga) ${ }^{3}$, elles prélèvent alors de la couche supérieure des contenants remplis (bouilloires et thermos) les prémices du thé au lait, que les Mongols appellent le « dessus » ou « meilleur » (deež). 
Debout, à l'extérieur de la yourte, les femmes offrent les prémices suivant le geste rituel d'"aspersion" (cacal/sacal): au moyen d'une cuiller (appelée du même nom cacal/sacal), elles prélèvent de la main droite dite "pure » dans la louche (šanaga) un peu des prémices de thé au lait et les jettent en l'air. Cette cuiller à asperger constitue un objet rituel et elle est pour cela rangée à part des autres couverts, sous une perche du toit de la partie féminine de la yourte.

Le liquide est projeté en l'air en direction des quatre orients ou en direction du Sud et l'aspersion est alors adressée à la terre et aux esprits-maîtres de la nature, ou encore en direction de la montagne, lieu de résidence des âmes des ancêtres qui surplombe le campement. Pour être offertes, les gouttes doivent non pas retomber sur le toit de la yourte, mais sur la terre, la terre des ancêtres.

10 Traditionnellement, l'aspersion matinale s'effectuait avec un aspersoir en bois en forme de cuiller rectangulaire à neuf cavités au manche parfois orné d'une tête de cheval sculptée.

11 Pour exécuter l'aspersion rituelle, les femmes doivent se couvrir la tête, règle élémentaire de respect, et se tenir debout, dos à la porte fermée, devant le seuil ou à droite du seuil, c'est-à-dire du côté sud-est, féminin, de la yourte.

Photo 2. Aspersion matinale des prémices du thé au lait

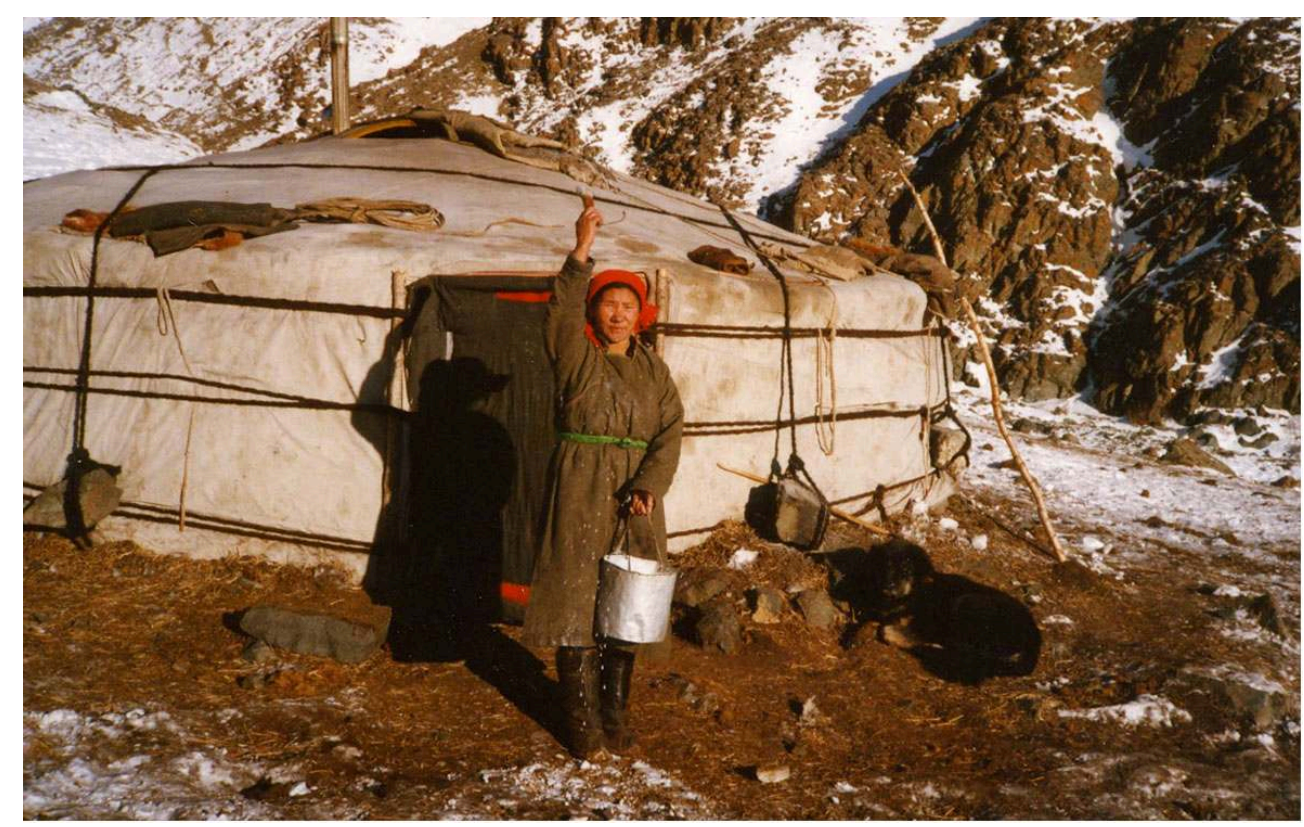

Grégory Delaplace (Uvs, 2000)

12 L'aspersion matinale consiste à faire se répandre le bonheur et la prospérité à la manière des gouttes éparpillées. Pendant qu'elles effectuent le geste d'aspersion, les femmes murmurent ou récitent mentalement des formules de bénédiction bouddhiques, des paroles d'appel (à soi) du bonheur, sous-entendu la bonne santé et la réussite des membres du foyer, ainsi que le bien-être du troupeau. Elles peuvent également s'excuser de fautes récemment commises. Le liquide aspergé est donc nécessairement un liquide blanc, blanc comme le lait, en l'occurrence du thé au lait. L'aspersion peut éventuellement se faire avec du «thé noir » (har caj), du thé ordinaire 
auquel on n'ajoute aucun aliment, mais le lait blanchit le thé et lui confère une qualité hautement propitiatoire.

Pour une action rituelle quotidienne, nous avons donc une association d'objets (louche et cuiller à asperger) et de gestes rituels (position et positionnement du corps, geste du bras et de la main, orientation de l'aspersion) toujours effectués de la même manière, au même endroit, au même instant de la journée, par les mêmes personnes (la gent féminine) et adressés à un même ensemble d'entités (esprits de la nature, mânes des ancêtres).

Les femmes qui doivent commémorer un mort décédé récemment (moins d'un an), versent dans une coupelle une partie des prémices du thé au lait avant d'effectuer l'aspersion rituelle - rarement après car le mort doit recevoir le dessus du "dessus » de l'offrande, il doit être le premier à recevoir les prémices du thé au lait. Parfois, les femmes oublient l'offrande de commémoration au mort; aussi, elles taisent cette omission ou feignent de l'ignorer pour que cela ne leur porte pas malheur. Remplie à bord avec « du dessus » (deež) des prémices, la « coupelle d'offrande [au mort] » (tahilyn cögc) est placée devant son portrait photographique sur l'autel domestique, au nord de la yourte (partie honorifique, masculine, de la yourte). Par ce geste, les femmes nourrissent l'âme du mort. Quant au contenu de la coupelle de la veille, il est préalablement ajouté aux prémices du thé au lait avant qu'elles ne soient offertes aux entités, à l'extérieur de la yourte. Rien ne se perd en matière de prémices, car elles sont garantes du bonheur des membres du foyer et de la prospérité du troupeau ${ }^{4}$.

Photo 3. Coupelle d'offrande au mort remplie des prémices du thé au lait

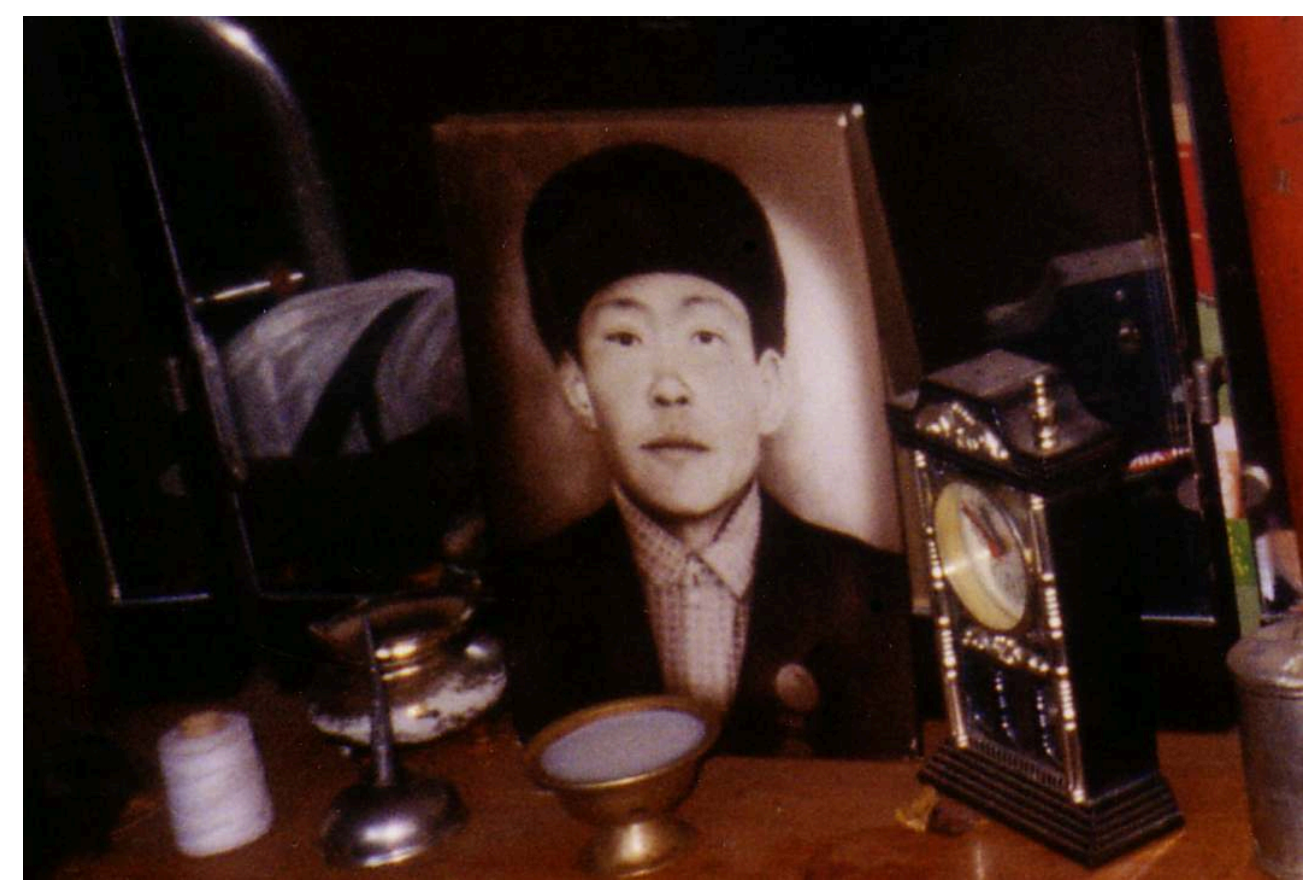

Sandrine Ruhlmann (Hentij, 2000)

\section{L'assiette d'hospitalité : les « dessus » et les « dessous » du lait}

Les journées des éleveurs sont scandées de visites. Dès l'entrée des visiteurs sous la yourte, avant même que les salutations ne soient prononcées, une assiette leur est 
systématiquement présentée. Cette assiette d'hospitalité se compose traditionnellement de laitages frais et gras sur le dessus, soit de la peau crémeuse (öröm) et du « beurre jaune » (šar tos) (beurre mou au goût rance, de couleur jaune), et de laitages séchés et maigres dans le fond, soit différentes sortes de fromages séchés (eezgij, aaruul, huruud). L'assiette est posée sur une table basse devant les visiteurs, hommes et femmes, qui prennent place dans la partie honorifique de la yourte.

Photo 4. Aération du lait chaud avec la louche (šanaga)

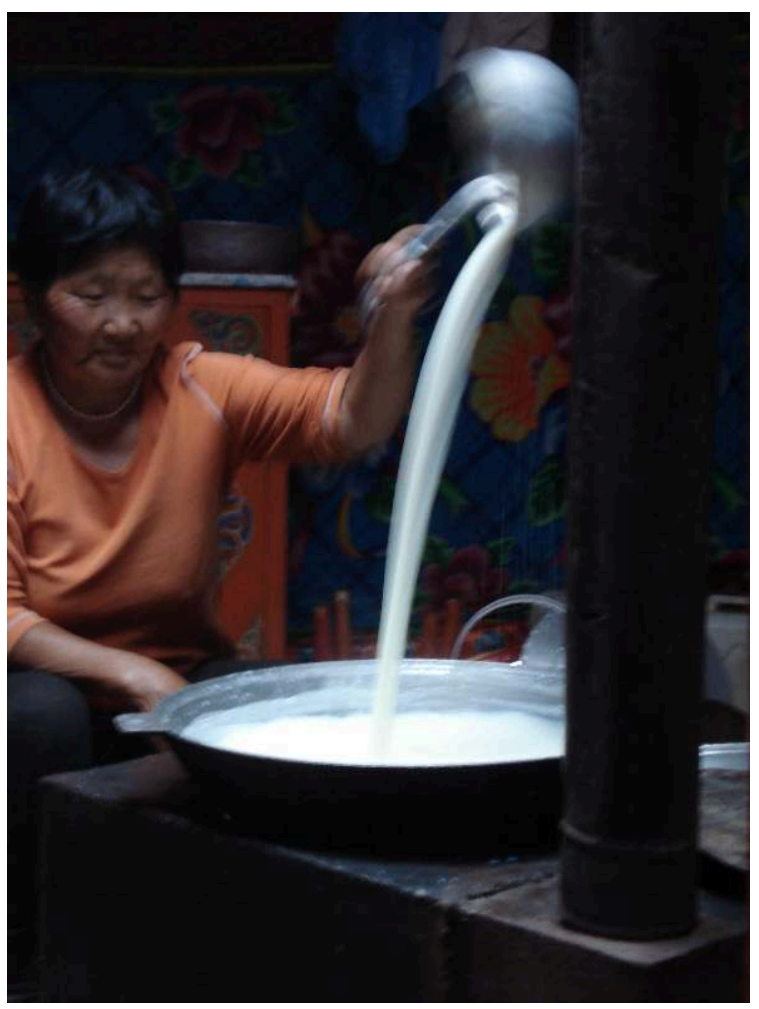

Sandrine Ruhlmann (Hentij, été 2003) 
Photo 5. Formation d'une mousse en surface

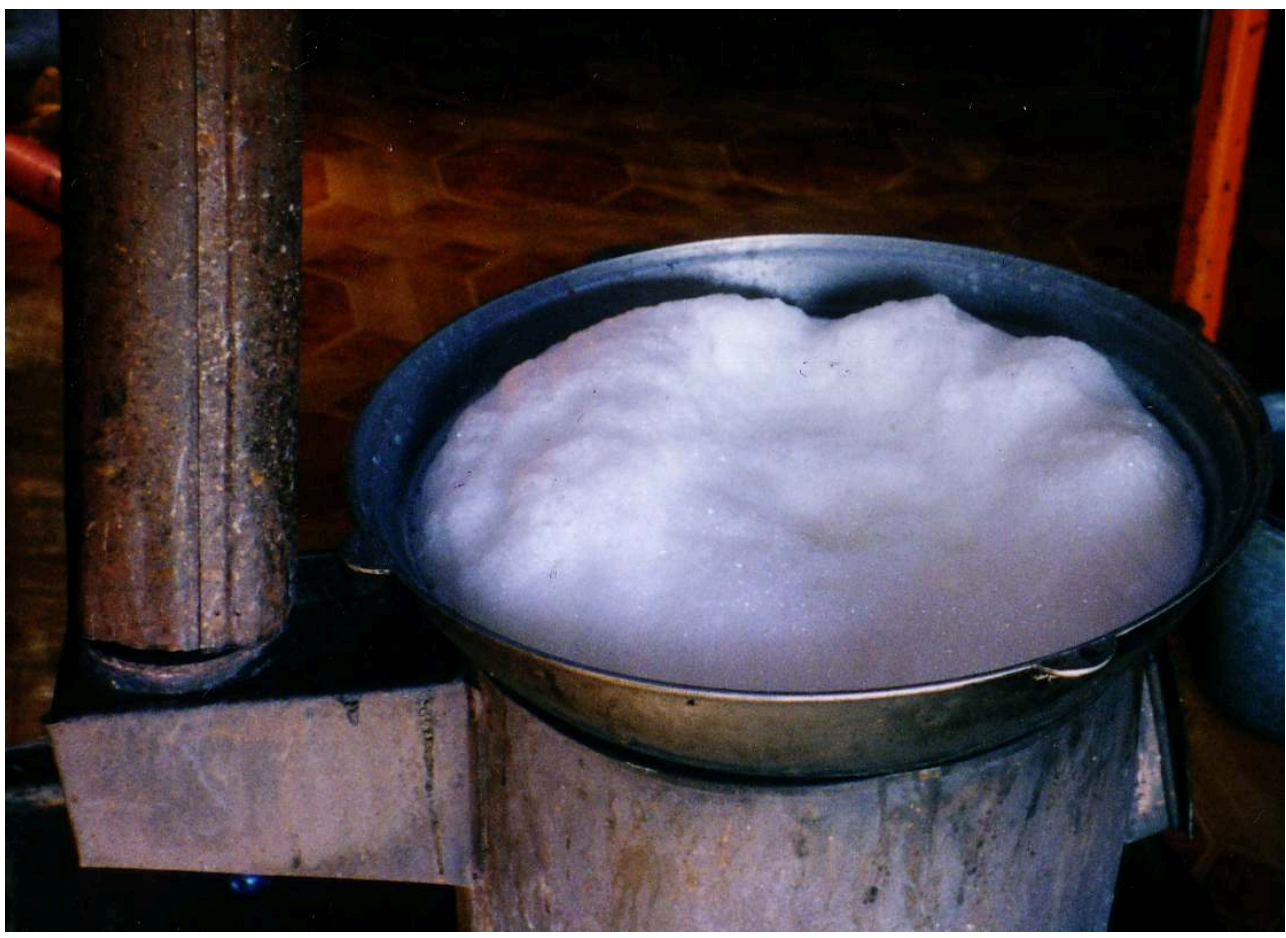

Sandrine Ruhlmann (Hentij, été 2003)

\section{Photo 6. Cuisson de la mousse}

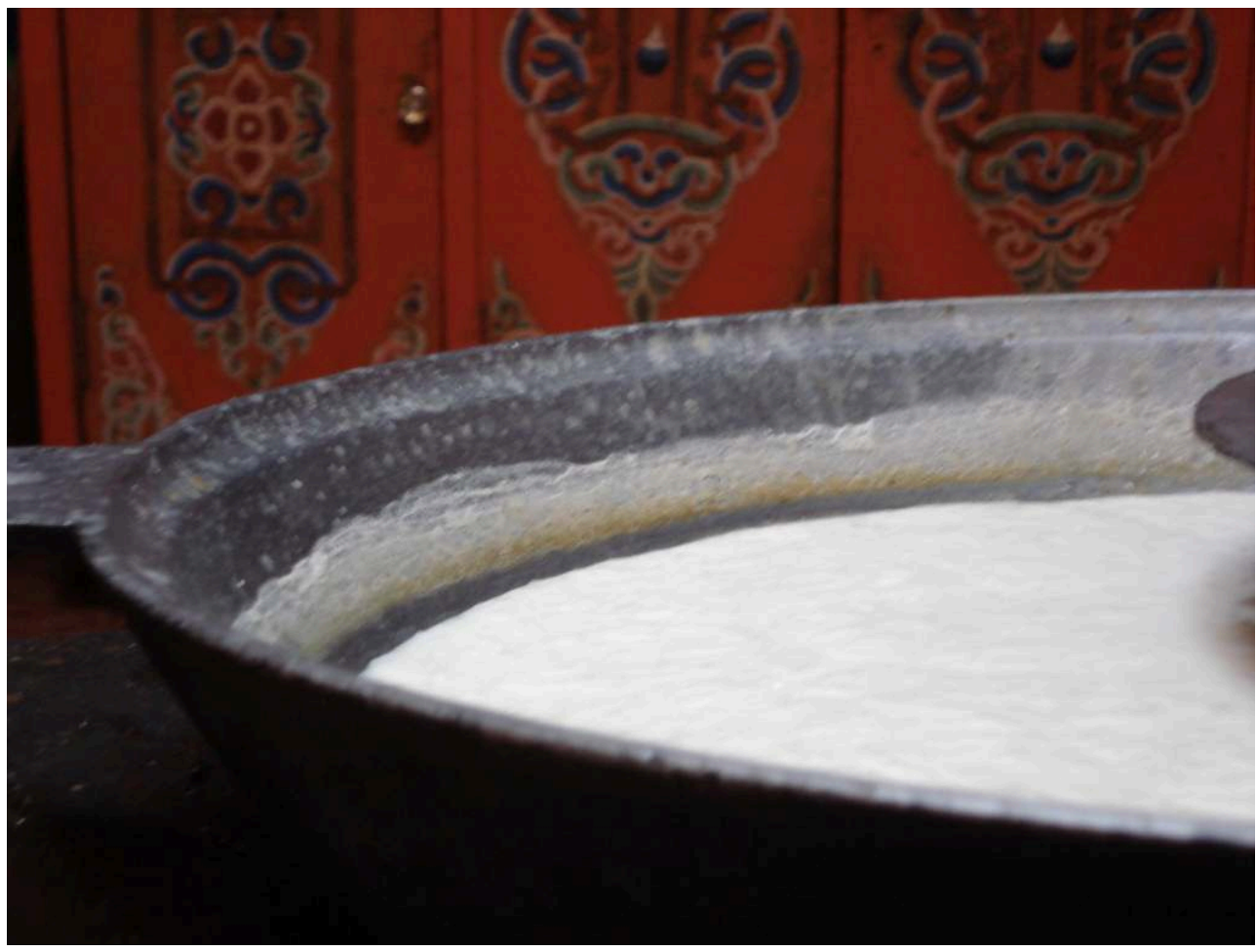

Sandrine Ruhlmann (Hentij, été 2003) 
Photo 7. Peau crémeuse du lait (öröm) séparée du lait

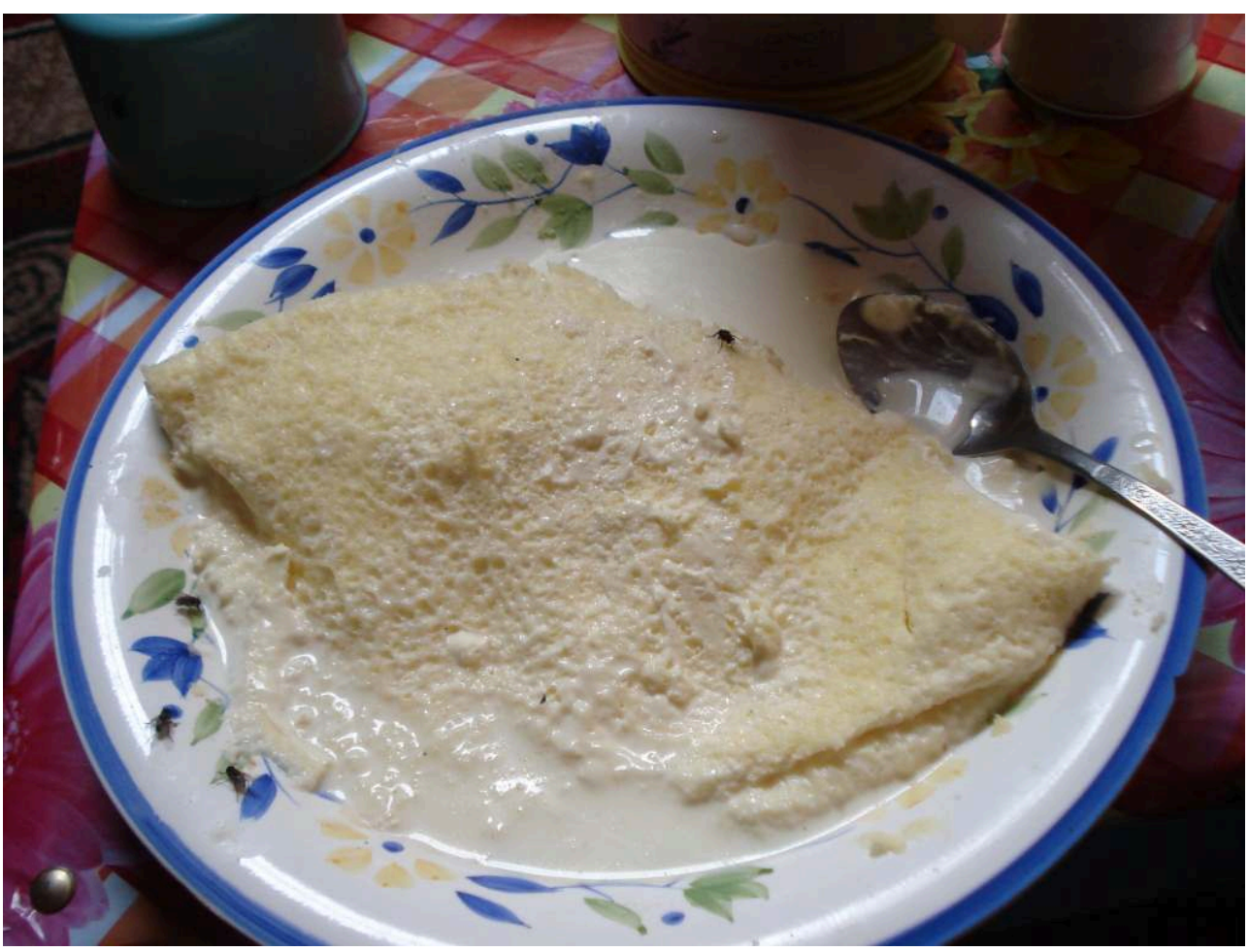

Produit laitier gras posé sur le « dessus » (deež) de l'assiette d'hospitalité

Sandrine Ruhlmann (Hentij, été 2003)

Les visiteurs se voient alors offrir un bol de thé au lait, et le réceptionnent, suivant le geste rituel d'offrande des aliments : la main droite "pure " tendue pour donner ou recevoir, le bras droit étant soutenu au niveau du coude ou de l'avant-bras par la main gauche, paumes tournées vers le ciel. Le bol rempli est maintenu entre le pouce sur son bord et les autres doigts sur sa base. En effectuant ce même geste, les visiteurs et l'hôtesse penchent légèrement le haut de leur corps en avant. Le mouvement des épaules, du torse et de la tête tout d'un bloc, ainsi que la position des bras et le geste de la main tendue, composent une salutation.

Les visiteurs sont tenus de ne prélever que des laitages honorifiques dit « du dessus » (deež). En les ingérant, ils s'imprègnent des qualités fastes de ces aliments incarnant la prospérité du troupeau et le bonheur des membres du foyer qui les offre. Cette offrande consommée est bénéfique aussi aux hôtes, qui tirent en retour les bénéfices de leur hospitalité. La prospérité des troupeaux est incarnée par l'abondance du lait et des laitages, la traite exprimant chez les peuples mongols le caractère faste du renouveau (Hamayon 1978, p. 219).

Quand les visiteurs sont sur le départ, la maîtresse de maison asperge de thé au lait la croupe des chevaux qui lui tournent déjà le dos. Cette aspersion rituelle est effectuée du même geste que l'aspersion matinale. Elle a pour objectif de souhaiter aux visiteurs qui s'apprêtent à traverser la steppe, qui est considérée comme un espace sauvage, une bonne route, une "route blanche » (cagaan zam). Cette aspersion peut également être effectuée avec du lait de jument fermenté (ajrag).

De nos jours, de nouveaux aliments viennent orner l'assiette d'hospitalité : des tranches de pain et des biscuits industriels. Ces farineux ne présentent aucune des 
caractéristiques valorisantes des laitages dits «du dessus » (deež), qui sont frais et gras et qui sont fabriqués à partir d'un aliment traditionnel, le lait. Ils garnissent donc le fond de l'assiette, et remplacent parfois les fromages séchés. Les visiteurs ne prélèvent aucun des aliments situés dans le fond de l'assiette, ces aliments « du dessous » (doož) que les hôtes consommeront au cours de la journée et des journées suivantes, en les trempant dans du thé.

Photo 8. Découpe des fromages

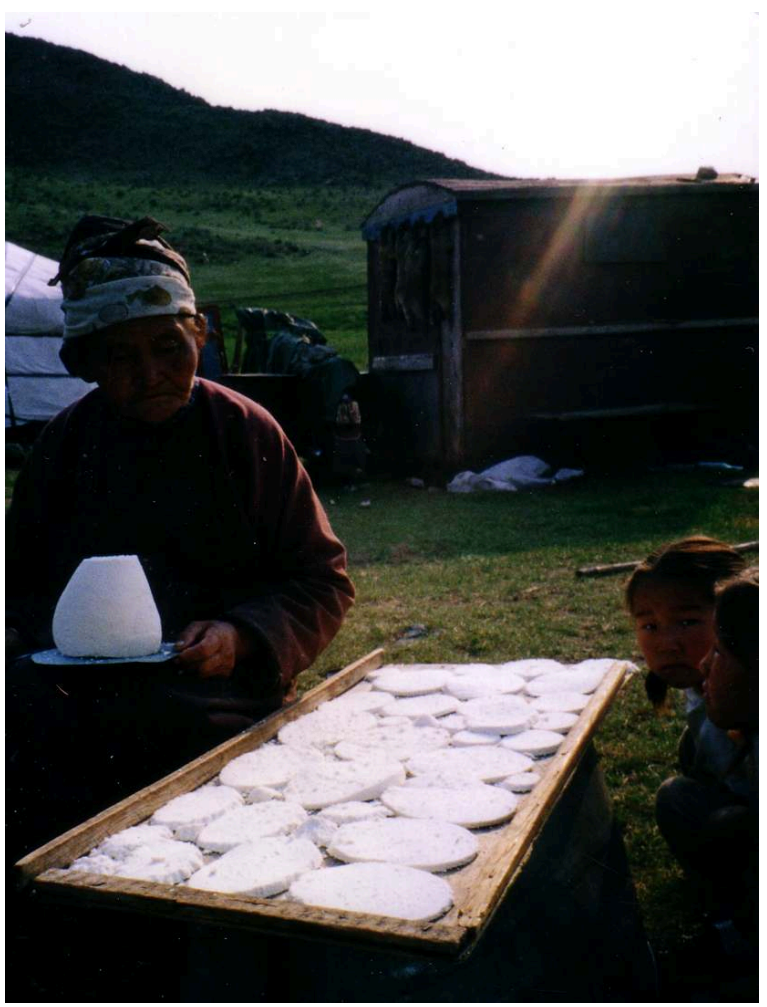

Sandrine Ruhlmann (Hentij, été 2003) 
Photo 9. Séchage des fromages au vent et au soleil

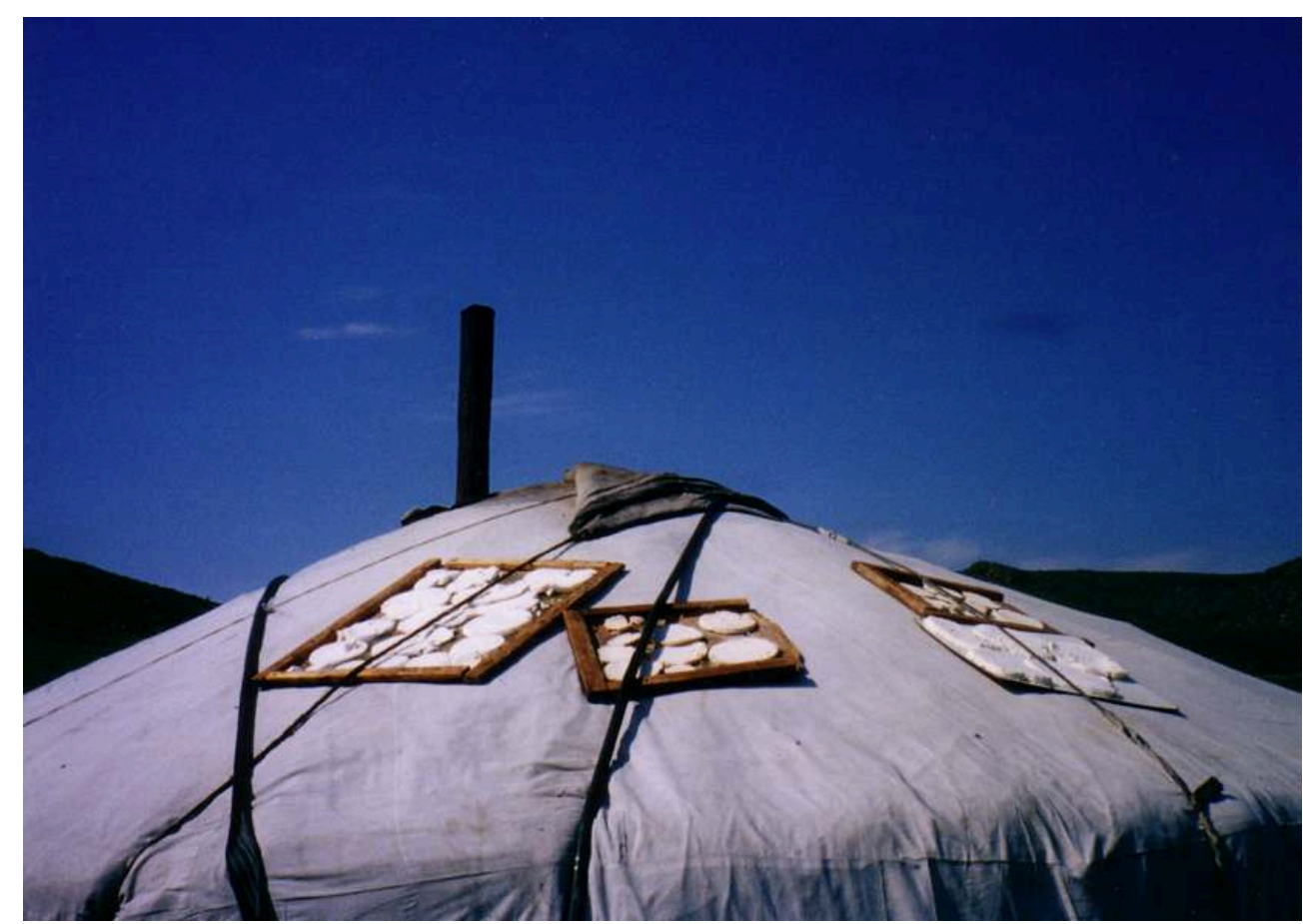

Produits laitiers secs posés dans le fond (doož) de l'assiette d'hospitalité

Sandrine Ruhlmann (Hentij, été 2003)

Nous retenons de l'étude de l'assiette d'hospitalité, traditionnelle ou revisitée par l'intégration d'une nouvelle catégorie d'aliments, les farineux, que les aliments « du dessus ", frais, gras, blancs, sont réservés aux visiteurs, aux membres extérieurs au foyer, tandis que les aliments « du dessous », séchés ou secs, maigres, que ce soient des laitages ou non, qu'ils soient blancs comme le lait ou non, sont destinés aux hôtes, aux membres du foyer. C'est ce que constatait déjà Roberte Hamayon (1975, p. 118) dans les années 1970 et qu'elle a représenté par un schéma losangique, indiquant que les Mongols destinent le séché à soi et le bouilli à autrui.

\section{Le Nouvel An lunaire: des produits laitiers sur le dessus}

21 Le Nouvel An lunaire est célébré à la fin de l'hiver, à une date calculée chaque année par les moines dirigeants du monastère Gandan, principal centre d'enseignement bouddhique situé à Ulaanbaatar. La fête du Nouvel An se déroule sur trois jours : le jour du grand ménage, le jour de fermeture de l'année et le jour d'ouverture de la nouvelle année. Le Nouvel An est une combinaison du mois et de la lune, ainsi que son appellation mongole le désigne, littéralement « mois blanc» (cagaan sar), le terme sar désignant la lune et le mois.

22 Autrefois, l'année débutait avec l'abondance des lactations, à la jonction de l'été et de l'automne. On rassemblait toutes les juments blanches du troupeau pour les consacrer, on jetait à terre les prémices du lait de jument fermeté (ajrag). Ceci explique que le lien entre la dénomination de la fête célébrant la nouvelle année et le lait soit «évident pour tout Mongol » (Hamayon 1978, p. 219). 

grandes fêtes célébraient le lait de jument fermenté, aux $4^{\mathrm{e}}$ et $7^{\mathrm{e}}$ lunes. Elles ont ensuite été fondues en une seule fête, marquant à la fois le début de la nouvelle année et la première consommation de lait de jument fermentés. C'est ce que confirme le récit de Marco Polo qui, invité à la cour de l'empereur Khubilai, assista à la "fête blanche » ou nouvelle année. Khubilai fit en effet adopter le calendrier chinois dans la deuxième moitié du XIII ${ }^{e}$ siècle, mais la date de la fête a subi ultérieurement des modifications. Aujourd'hui, elle est célébrée à la nouvelle lune du premier mois de l'année. Pour associer la nouvelle année à la prospérité et au renouveau originellement incarnés par les produits laitiers, les Mongols conservent à l'automne des quantités de lait et de produits laitiers frais et séchés pour le repas du « mois blanc».

un premier temps, les familles procèdent à un grand ménage, comme pour balayer l'année écoulée et laisser la place à une nouvelle année. Puis, le soir du réveillon dit « soir fermé » (bitüün oroj), à l'heure où le ciel se ferme (le soleil se couche) et quand chacun a fermé son corps (boutonné ses habits de fête et fermé son corps par le port d'un chapeau ou d'un foulard), les familles du campement s'appliquent à bien fermer l'ancienne année en s'offrant et en consommant des nourritures "fermées" (bitüï). La queue grasse du mouton, partie fermant le corps de l'animal sur pied, constitue une part de choix du plat traditionnel de mouton entier bouilli (šü̈s), qui est considéré comme un plat «noir », puisque la viande est consommée sans aucun autre aliment l'accompagnant. En ce «jour fermé » (bitüün ödör), ce plat de viande festif par excellence est appelé «fermeture» (bitüüleg), terme formé sur la racine du verbe «fermer» (bitüüleh). Enfin, des raviolis (buuz) qui, ce même jour, constituent des «nourritures fermées » (bitüün hool), parce qu'ils sont considérés en fonction de leur garniture exclusivement carnée (donc noire) qui est enfermée dans une enveloppe de pâte de farine de blé. L'année se ferme par la consommation de plats carnés, noirs, par opposition à la nouvelle année qui s'ouvre avec la consommation de plats lactés, blancs.

Le dernier jour de fête est le jour d'ouverture de la nouvelle année, c'est le «premier nouveau jour » (šine negen ödör). De ce jour en particulier dépend tout ce qui adviendra au cours de l'année. Ainsi, les familles favorisent le bon déroulement de l'année en offrant à manger des " aliments blancs ", fastes, incarnant la prospérité et le bonheur, à un grand nombre de visiteurs extérieurs au campement (essentiellement des hommes, mais aussi des femmes et des enfants). Au petit matin, les familles décongèlent des « aliments blancs » frais et gras : de la peau crémeuse du lait (öröm), du «beurre blanc » (cagaan tos) $)^{6}$, du « beurre jaune » (šar tos) et du fromage frais (bjaslag). Puis, une grande quantité de yaourt (tarag) et de lait (sü̈) pour préparer une bouillie épaisse et sucrée de riz et de raisins secs - bouillie de couleur blanche appelée "cagaa », le terme cagaan désignant quant à lui la couleur blanche. Les femmes montent alors des pyramides de gâteaux en forme de semelle (ul boov); elles déposent des fromages séchés à leur base, sur le «dessous » (doož), et des laitages frais et gras à leur sommet, sur «le dessus » (deež). Une pyramide de gâteaux-semelle est placée sur la table basse, au nord-ouest de la yourte, une autre pyramide est disposée sur l'autel domestique. À ces "aliments blancs" s'ajoutent des raviolis qui sont considérés, en ce jour d'ouverture de la nouvelle année, non pas en fonction de leur garniture, mais de leur enveloppe de pâte de farine blanche, associée au caractère faste de la couleur des laitages. Cette association leur confère la valeur symbolique de renouveau et de prospérité des

Études mongoles et sibériennes, centrasiatiques et tibétaines, 43-44 | 2013 
produits laitiers frais et gras ${ }^{7}$. Le référent absolu est le lait et sa couleur blanche, et lui sont associés voire assimilés les propriétés, qualités et symboles qui leur sont rattachés.

Photo 10. Pyramide de gâteaux-semelle

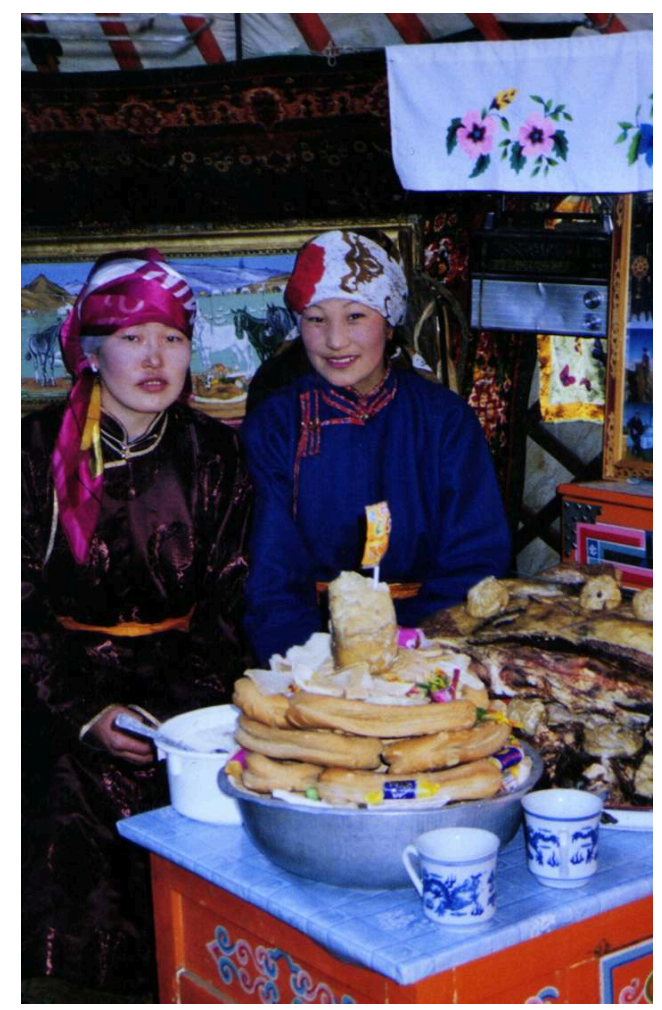

Pour cette pyramide, de haut (dessus, gras) en bas (dessous, sec) :

- «beurre blanc » (cagaan tos)

- peau du lait (öröm)

- fromages séchés (aaruul, huruud)

- gâteaux-semelle (ul boov)

- fromages séchés (aaruul, huruud)

Sandrine Ruhlmann (Hentij, 2000)

Pour les Mongols, l'offrande et la consommation des « aliments blancs » les rappellent à leur histoire, celle de leurs ancêtres et celle, glorieuse, de l'empire mongol. Ils relient aujourd'hui l'image de Chinggis Khan au mode vie nomade des éleveurs, à leur habit et à leur alimentation traditionnels. Aujourd'hui, le gouvernement et les intellectuels mongols élèvent l'empereur Chinggis au rang de symbole identitaire national, et avec lui la consommation très répandue de la boisson emblématique de son époque, le lait de jument fermenté (témoignage de Plan Carpin 1965, pp. 48-49). Les boissons, regroupées sous un terme générique (umda [unda]), étaient faites à partir du lait fermenté de vache, de chèvre ou de jument qui était additionné de lait ou coupé d'eau (Even \& Pop 1994, p. 88, § 124 et note 67) ${ }^{8}$. Racontant son séjour à la cour de Khubilai Khan, Marco Polo décrit en détail la traite annuelle de juments blanches à l'occasion de la "fête blanche », dans le chapitre 62 intitulé " De la grande fête que fait le grand khan le jour de l'an " (1988, pp. 161-164). Sa description met en relief l'importance de l'usage de la couleur blanche qui était «bonne et prometteuse de bonheur». Le Khan et ses sujets s'habillaient en robe blanche, recevaient des présents de couleur blanche, faisaient venir plus de cent mille chevaux de race blancs en cadeau au Khan et se souhaitaient bonheur et prospérité. La célébration de la nouvelle année s'exprimait par un usage 
généralisé de la couleur blanche au XIII ${ }^{e}$ siècle, et cet usage prédomine encore aux Xx ${ }^{e}$ et xxI ${ }^{\mathrm{e}}$ siècles à la fête du Nouvel An.

Nous retenons que, dans le contexte actuel du Nouvel An, le choix des familles porte sur la couleur blanche, en l'occurrence sur les traditionnels "aliments blancs" et désormais aussi sur des farineux, qui prennent une place considérable parce qu'ils sont de couleur blanche et/ou parce qu'ils sont offerts par multiple de trois, chiffre faste chez les Mongols bouddhistes ${ }^{9}$. Les farineux sont, dans ce contexte précis, associés aux " aliments blancs » et les familles leur confèrent les mêmes propriétés. Cependant, le sens accordé à la couleur blanche a évolué en différentes significations associées dans le cadre des rituels de naissance et de mort.

\section{La naissance et la mort : évolutions de la symbolique des produits laitiers et du lait}

La naissance est un événement heureux, et cependant caché pour ne pas exposer le corps du nouveau-né aux âmes errantes et esprits affamés qui s'y logeraient en vue d'y être nourris. Le sang étant considéré comme une substance impure par le clergé bouddhique, ce dernier n'intervient pas dans les rites de naissance biologique. D'ailleurs, les femmes accouchent depuis les années 1950 à l'hôpital et ce sont les sagesfemmes qui se chargent du traitement du placenta et du cordon ombilical, aujourd'hui jetés ou utilisés dans la confection de cosmétiques ${ }^{10}$. La religion bouddhique imprègne par contre les rites de la naissance sociale du nouveau-né : dation du nom, toilette et emmaillotage, ainsi que le repas qui accompagne le rituel de la toilette (Ruhlmann 2010).

29 Le matin du retour de l'accouchée et du nouveau-né sur le campement, la mère de l'accouchée fait une aspersion de thé au lait en tournant trois fois autour de la yourte dans le sens du soleil, pour favoriser leur bon retour. Elle prépare ensuite pour tous les membres du campement une bouillie (cagaa) de riz cuit dans du yaourt additionné d'un peu de farine et de sucre en poudre. Des variantes de cette bouillie existent : les femmes peuvent ajouter du beurre, différentes sortes de fromages séchés (eezgij, aaruul ou huruud) préalablement réduits en poudre, etc. La consommation de cette bouillie blanche avant l'arrivée de l'accouchée est une marque de respect et souhaite la bienvenue au nouveau-né. C'est littéralement « la fête d'être avec un nouvel être » (šine hüntej bolsny bajar).

Pour autant, personne ne visite l'accouchée et le nourrisson pendant un mois. Ces deux êtres sont fragiles: la mère a les os disloqués pour permettre l'accouchement, le nourrisson n'a pas encore les os soudés et son corps n'est pas fortifié. Ils sont momentanément isolés par mesure de protection. Pendant cette période de confinement, la mère est contrainte à un régime maigre pour rétablir l'équilibre de son corps échauffé par l'accouchement et souillé par le sang. Ainsi, elle ne mange pas de produits gras, comme la peau crémeuse du lait (öröm) et le «beurre jaune » (šar tos), et boit de préférence du « thé noir » (har caj).

31 Un mois après la naissance, la toilette rituelle met un terme au confinement: le nourrisson est « lavé » avec un bouillon de viande gras et fortifiant appliqué sur sa peau au moyen d'un torchon imbibé, et la mère partage le repas de fête avec sa famille et celle du père. La consommation du plat de viande - un mouton abattu pour l'occasion - s'accompagne d'un bol du bouillon de cuisson de la viande, qui comporte le gras de la 
chair et la graisse de l'os. Ce bouillon gras va « lier » les os de la mère et du nourrisson et fortifier leur corps. La mère consomme en outre une part de viande constituée de trois vertèbres cervicales qui a la propriété, dit-on, de « rendre fort $»^{11}$.

Le repas de célébration de la naissance commence et finit impérativement par une consommation de thé au lait et d'une bouillie épaisse et sucrée de riz et de différents laitages que les femmes associent à leur convenance. Des produits laitiers frais et séchés sont présentés dans des grands plats et chaque convive est tenu de se servir copieusement et d'en rapporter chez soi. La présence des « aliments blancs » marque la fin du régime maigre de l'accouchée et du nouveau-né. Leur consommation par l'accouchée et la famille purifie les deux corps jusque-là souillés, concrétise la resocialisation de la mère et la socialisation du nouveau-né et les place sous de bons augures. À cet instant, le nouveau-né incarne le bonheur, la prospérité et la vie: le renouveau présentement associé à la croyance chamanique en la renaissance des âmes des morts dans le corps des nouveau-nés.

33 Les "aliments blanc » et le blanc prennent un autre sens dans le cadre des pratiques funéraires. Événement considéré comme étant néfaste par le clergé bouddhique, la mort d'un humain est dissimulée par les membres de la famille, qui effectuent divers rites d'inversion de l'ordinaire pour protéger l'âme de leur mort que des âmes errantes affamées dévoreraient volontiers. Considérant le corps du mort comme étant une souillure majeure, les moines ne s'investissent pas dans les traitements physiques du cadavre $^{12}$. La famille se charge donc d'enterrer ses morts, en suivant les règles édictées par la loi gouvernementale de 1955.

34 La famille effectue des purifications externes et internes faisant intervenir l'utilisation et la consommation des "aliments blancs». Avec un mélange d'eau et de lait, les femmes lavent le cadavre et la yourte où il a séjourné avant d'être mis en bière et enterré. Avec ce même mélange blanc, les membres du cortège funéraire se lavent les mains et le visage à leur retour du cimetière, pour se purifier du contact avec le corps du mort. Enfin, pour blanchir l'âme du défunt, la famille offre une multitude de nourritures «blanches » aux membres de la famille étendue, aux voisins et à tous les visiteurs venus présenter leurs condoléances (Ruhlmann 2009a).

Pour clore la période de deuil de 49 jours, pendant laquelle les membres de la famille restreinte du mort sont socialement isolés (ils ne travaillent pas et ne reçoivent aucune visite), un repas est offert aux enfants de la famille et du voisinage ${ }^{13}$. Ce repas est composé uniquement d'" aliments blancs ", contrairement aux trois repas funéraires offerts les $3^{\mathrm{e}}, 7^{\mathrm{e}}$ et $21^{\mathrm{e}}$ jours après l'enterrement, qui comprennent notamment une soupe de viande - mais d'une viande exceptionnellement maigre ${ }^{14}-$ et de farineux associés cette fois encore aux « aliments blancs ».

36 Le repas de clôture du deuil comprend des «aliments blancs " pour mettre fin à la période noire de deuil. C'est le repas des « dix mérites blancs» (arvan cagaan bujan) : thé au lait, bouillie sucrée de lait et de riz, soupe de thé au lait avec millet et petits raviolis de viande grasse, bouillie de lait et de yaourt, «beurre blanc ", bonbons, morceaux de sucre, gâteaux-semelle, différentes sortes de fromages séchés, etc. Ce repas est consacré aux enfants qui représentent par analogie les nouveau-nés dans lesquels les âmes des morts sont destinées à renaître. Les enfants rapportent chez eux un à trois gâteaux-semelle, des morceaux de sucre et des portions de «beurre blanc». Les " aliments blancs» et les aliments de couleur blanche offerts et consommés sur place ou rapportés à la maison par les enfants permettent de clore et de supprimer la période 
de deuil, de purifier les corps souillés par le contact avec la mort et de rappeler (à soi) le bonheur en effectuant des actions méritantes. La notion de "mérites" (bujan) s'applique aux offrandes de nourritures blanches, produits laitiers ou aliments de couleur blanche, ou considérés comme de couleur blanche comme les gâteaux et autres farineux. Les «mérites ", appelés également « mérites blancs » (cagaan bujan), sont des offrandes présentes en diversité (grande variété d'aliments) et en quantité (multiplication de chacun d'eux par leur découpe ou leur constitution), comme les divers produits laitiers offerts et comme les nombreux grains de riz ou de millet qui entrent dans la composition des bouillies de laitage.

Les «mérites » accumulés ont la propriété d'appeler le bonheur à soi et de rétablir le bonheur perturbé par un événement souillant. Ils incarnent le renouveau et la prospérité au même titre que les « aliments blancs ».

Il semblerait que progressivement, et peut-être à partir des années 1990, quand le bouddhisme est reconnu religion officielle de l'État après avoir été réprimé par le gouvernement communiste, et que le chamanisme est une religion reconnue et autorisée, la symbolique des produits laitiers a évolué. Parce qu'ils sont de couleur blanche, les produits laitiers sont devenus une substance purificatrice et ont remplacé l'agent de purification mongol de référence - le feu - dans les rituels qui exigent une purification ${ }^{15}$. Désormais, dans le cadre du rituel funéraire notamment, les Mongols ne purifient plus les personnes et les objets en les faisant passer entre deux feux ou audessus d'un feu, comme a pu en témoigner Plan Carpin au XIII siècle (1965, pp. 40, 43). Les objets, les corps, les âmes et les personnes sont purifiés en les lavant avec un mélange de lait et d'eau ou en les aspergeant de ce mélange [purification externe], et en consommant des «aliments blancs» ou des aliments de couleur blanche qui leur sont associés dans cette fonction [purification interne] et qui, offerts et consommés en multitude, constituent des "mérites» (Ruhlmann 2009a). Offrir des "mérites» constitue une action méritante au sens bouddhique du terme, qui favorise la bonne renaissance de l'âme après la mort, c'est-à-dire dans un humain et non dans un animal. Les notions bouddhiques de purification et de mérites sont toutes les deux rattachées à des usages efficaces d'aliments "blancs", des produits laitiers et des produits de couleur blanche tous manipulés, offerts et/ou consommés en diversité et en multitude. De fait, des pratiques purificatrices et propitiatoires sont aujourd'hui associées, voire confondues en une seule et même action dans certains contextes, et donc la couleur blanche se trouve associée aussi à la purification, tandis que les aspersions avec du lait ou du thé au lait sont par nature des propitiations.

\section{Le lait de jument fermenté et l'alcool de lait distillé, des boissons emblématiques}

Les deux boissons alcooliques sont traditionnellement fabriquées par les hommes, qui se tenaient du côté masculin ouest à l'extérieur de la yourte pour le lait de jument fermenté (ajrag), dans la partie honorifique nord de la yourte pour l'alcool de lait distillé (arhi). De nos jours, les juments sont très souvent traites par les femmes, et les hommes - du campement ou en visite - sont chargés de battre le lait fermenté dans une outre en peau ou dans un haut baril en bois ou en plastique ${ }^{16}$. Les femmes sont aujourd'hui mises à contribution dans la chaîne de distillation du lait et recueillent le 
lait distillé non pas au nord-ouest comme les hommes le font, mais au sud-est, ce qui nécessite de modifier l'orientation de l'alambic.

La consommation des boissons alcooliques est ritualisée et spécifique à chacune d'entre elles. Ce sont les hommes qui les offrent. Le lait de jument fermenté se boit dans un grand bol collectif, de la main droite ou des deux mains. En le présentant des deux mains portées à son front ou de la main droite soutenue au niveau du coude par la main gauche, l'hôte le fait circuler à ses visiteurs qui le reçoivent chacun à leur tour d'un même geste et qui ne boivent que quelques gorgées. L'hôte remplit le bol quand il lui est rendu pour que chaque visiteur boive « du dessus » (deež). À l'inverse, l'alcool de lait distillé se boit collectivement mais dans des petits bols individuels que chacun est tenu de vider. Cette boisson est offerte d'une seule main, de la main droite " pure » soutenue de la main gauche au niveau de l'avant-bras, et réceptionnée de la même manière. Le visiteur doit boire tout le contenu de son petit bol et pas uniquement « du dessus ».

Photos 11 \& 12. Offrande-circulation des bols d'ajrag et d'arhi (ici, de la vodka industrielle)

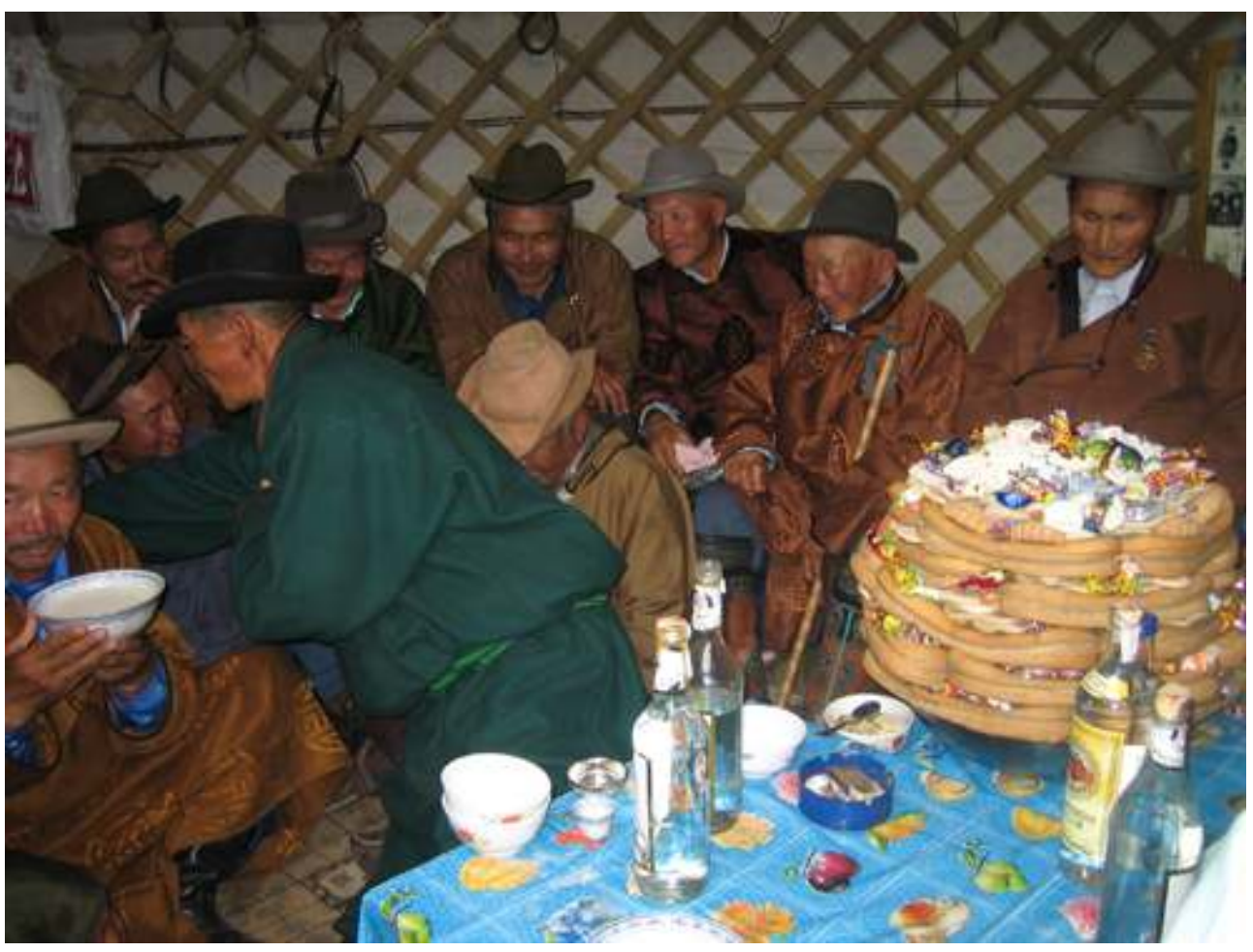

L'homme au centre (manteau vert) fait le service; on peut voir sa main gauche soutenir son coude droit pour offrir solennellement un bol d'ajrag

Linda Gardelle (2006) 


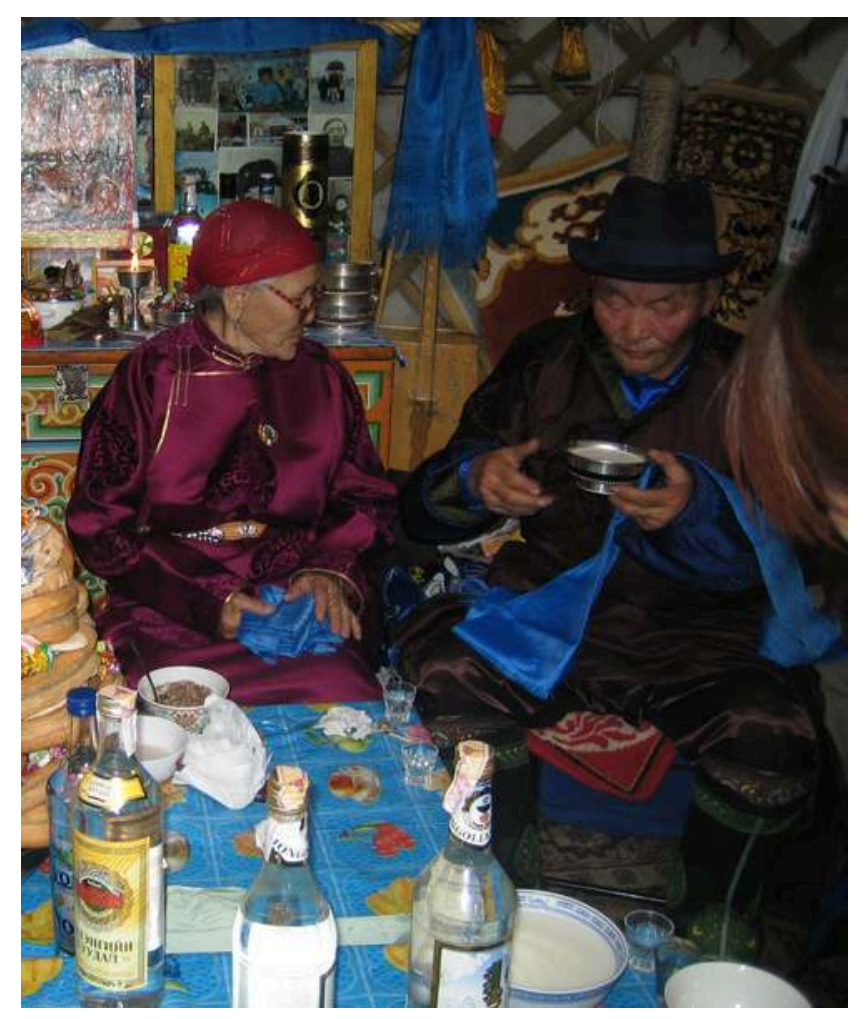

Linda Gardelle (2006)

41 D'une manière générale, le lait de jument fermenté se consomme en grande quantité à la période estivale et sa consommation est liée aux grands rassemblements et aux fêtes. Le cheval bénéficiait d'une place d'honneur très large parmi les animaux élevés. Cet animal de monte fut l'atout majeur des empereurs mongols au XIII ${ }^{\mathrm{e}}$ siècle. Chez les Mongols de Sibérie, il était par ailleurs l'animal sacrifié lors des sacrifices collectifs (Rintchen 1977-2) - le mouton l'était lors des sacrifices privés (Hamayon 1990, pp. 637-638). Enfin au XIII ${ }^{e}$ siècle et jusqu'à la fin XVI ${ }^{e}$ du siècle, date de conversion de l'aristocratie mongole au bouddhisme, le lait de jument fermenté constituait la boisson la plus répandue. Elle désaltérait et soignait, elle était la boisson rituelle, la boisson sacrificielle, la boisson de commémoration des morts, la boisson offerte aux mânes des ancêtres gengiskhanides, et des juments blanches étaient sacrifiées sur les tombes des empereurs et enterrées dans les tombes des hommes de haut rang afin qu'ils en soient pourvus dans l'au-delà ${ }^{17}$.

$42 \mathrm{Au}$ XIII ${ }^{\mathrm{e}}$ siècle, comme l'attestent certains passages de l'Histoire secrète des Mongols (Even \& Pop 1994, § 85, 124), manger revenait à manger de la viande avec son bouillon de cuisson et à boire du lait de jument fermenté ${ }^{18}$. Le lait de jument fermenté constituait plus qu'une boisson quotidienne : produit en très grande quantité à partir du lait de juments blanches rapportées de tout l'empire, il constituait la boisson cérémonielle par excellence et exprimait le caractère faste d'une fête (Even \& Pop 1994, § 130, 154, 187, 189). Depuis la modification de sa date de célébration, il semblerait que la fête du Nouvel An célèbre davantage la nouvelle année que la production de lait de jument fermenté - celui-ci étant de plus en plus rarement au menu - et la reprise de lactation, ce d'autant plus que la fête du premier lait de jument fermenté subsiste. Cette fête du premier ajrag de l'année dite "prendre la jument» (güü barih) se célèbre en juin (Bianquis-Gasser 1996). La traite continue des juments et l'abondante production 
quotidienne de lait de jument fermenté (des Mongols disent que certains hommes boivent jusqu'à 15 litres d'ajrag en une journée) célèbrent le renouveau, non plus en association avec la nouvelle année, mais avec la prospérité et la bonne santé du bétail ${ }^{19}$.

Aujourd'hui, c'est également à l'occasion de la fête nationale du Naadam ${ }^{20}$, célébrée à la mi-juillet, que les Mongols fabriquent et consomment de grandes quantités de lait de jument fermenté. Pour commencer les festivités, qui ont lieu du 11 au 13 juillet, des aspersions de lait de jument fermenté sont faites aux quatre orients. Les chevaux sont à l'honneur : les courses évoquent les chevauchées des cavaliers de l'empire mongol. Les trois ou cinq chevaux gagnants sont aspergés de lait de jument fermenté au niveau de leur tête et de leur croupe. Les lutteurs qui gagnent au combat s'abreuvent d'une louche de lait de jument fermenté et l'ultime gagnant s'en voit offrir cérémonieusement un grand bol. C'est aussi de lait de jument fermenté que les Mongols aspergent leurs chevaux quand ils se dispersent après s'être rassemblés pendant les trois jours de fête.

$\mathrm{Au}$ Nouvel An, et dans toutes les occasions où l'on consomme du lait de jument fermenté (ajrag) ou de l'alcool de lait distillé (arhi) - ou désormais de la vodka, sa variante industrielle éponyme -, il est de coutume d'en offrir quelques gouttes à l'esprit-maître du feu en aspergeant le fourneau, ainsi qu'au ciel en aspergeant en direction du ciel. Ce sont souvent les hommes, éventuellement l'homme le plus âgé, qui procèdent à l'aspersion rituelle d'une chiquenaude de l'annulaire droit imbibé d'alcool de lait.

Nous constatons que les Mongols accordent à ces deux boissons une importance rituelle dans la célébration des fêtes calendaires. Cependant la fabrication et la consommation successives du lait de jument fermenté sur une période réduite en été lui confèrent un statut particulier de produit rare et prisé. Sa fabrication évoque le renouveau, sa consommation célèbre la fête et le rassemblement des humains. Si auparavant cette boisson incarnait le rituel de la première traite annuelle des juments, elle incarne avant tout aujourd'hui la célébration de la fête nationale du Naadam. Et si chez les Mongols médiévaux, elle était une boisson réservée en priorité à l'aristocratie guerrière, elle est aujourd'hui une boisson du peuple, symbole de l'identité nationale liée à l'emblématique image de l'empereur Chinggis.

\section{Le lait, une matière sociale, économique et politique}

La Mongolie a longtemps été un pays tampon au cœur des conflits et des enjeux économiques et politiques entre l'URSS et la Chine. Après soixante-dix années de tutelle soviétique, la Mongolie a repris en main son histoire et s'est réapproprié ses traditions et sa culture, tout en s'intégrant, à partir de 1990, au marché économique mondial. La fin du collectivisme et la privatisation du bétail sont allées de pair avec une dégradation du niveau de vie des éleveurs qui étaient auparavant bien soutenus par l'État. Depuis la chute du Mur de Berlin et le démantèlement de l'URSS, la Mongolie connaît une évolution accélérée, laissant les éleveurs nomades seuls face à de nombreuses difficultés d'ordre climatique et économique. Mais le secteur de l'élevage représentant un tiers du Produit Intérieur Brut (PIB) du pays, les autorités mongoles ont pris conscience, depuis quelques années, que le pastoralisme est non seulement le meilleur moyen de tirer bénéfice des immenses territoires arides du pays, mais qu'il représente aussi un cas exemplaire de production écologique. L'exemple de la Chine 
voisine, où les élevages intensifs ont donné naissance à de nombreuses maladies touchant les animaux ainsi que les populations humaines, et l'intérêt accru d'une certaine partie de la population mondiale pour les produits sains et écologiques, ont fait comprendre aux hommes politiques mongols qu'ils avaient intérêt à encourager l'élevage nomade. Le lait est ainsi à la fois un aliment relevant d'enjeux sociaux, écologiques, économiques et politiques.

\section{À système pastoral, système alimentaire}

47 Les aspects sociologiques des produits laitiers sont étroitement liés à leur mode de fabrication et de conservation (Ruhlmann 2013a), et les manières d'offrir et de consommer les « aliments blancs » nous font comprendre dans quelle mesure le lait est une matière sociale. Les produits gras et frais sont réservés aux visiteurs, aux personnes à respecter comme les aînés, c'est-à-dire les hommes et les personnes âgées, et aux âmes des morts récents, aux divinités, aux mânes des ancêtres selon les situations et les rituels. De la même manière et inversement, les produits maigres et secs sont destinés aux membres de la famille et aux cadets, c'est-à-dire les femmes et les enfants.

Le lait demande un savoir-faire tout aussi varié et détaillé, plus large et tout aussi complexe que le savoir-faire relatif à la viande. Il faut traire le bétail, stocker le lait, le faire bouillir et le filtrer, l'aérer, le transformer suivant diverses techniques en un grand nombre de différents dérivés gras et frais, pour la consommation immédiate, séchés et maigres, pour une consommation différée. À la fin de l'automne, il s'agit de conserver une importante quantité de lait frais à l'état congelé, suivant une congélation spontanée au contact des températures hivernales, pour en disposer en grande quantité au repas du Nouvel An. La viande, quant à elle, demande de connaître la méthode d'abattage et les techniques de débitage, la cuisson étant la même quel que soit le plat préparé : les morceaux de chair découpés, consommés sous forme de soupe, et les parts de viande rattachées à l'os, et consommées sur l'os avec un couteau, sont bouillis. Enfin, la viande est consommée quotidiennement certes, mais en très petite quantité, comparée aux laitages qui sont toujours préparés et consommés en abondance, que ce soit l'été ou l'hiver, au Nouvel An, lors de la célébration d'une naissance, d'un mariage ou d'une mort. La viande et les laitages sont des aliments qui «nourrissent » au sens mongol du terme. Si la viande et les produits laitiers constituent les deux catégories d'aliments traditionnels et socialement valorisés, il apparait que les laitages prennent une part plus importante dans les rituels et la couleur blanche a une signification forte, étendue et aujourd'hui multiple. C'est parce que, depuis la conversion au bouddhisme, les "aliments blancs» sont rattachés aux notions bouddhiques de purification et de mérites et associés à une catégorie d'aliments jusquelà méconnue et dépréciée des peuples mongols, les farineux (Ruhlmann 2013b). Les farineux donnent une consistance à la soupe ordinaire, mais ne font pas repas. Malgré tout, certains d'entre eux, comme les raviolis et les gâteaux-semelle, sont érigés au même rang que les produits laitiers dans le cadre des rituels de la vie biologique et sociale. 


\section{Du gouvernement aux éleveurs}

49 Le regain d'intérêt pour les produits laitiers traditionnels, compte tenu de leur importance sociale et culturelle, se ressent à travers les actions du gouvernement qui, à la fin de la décennie 1990, entend réapprovisionner les étalages des marchés et magasins alimentaires des villes et notamment de la capitale en produits laitiers mongols. Il était en effet jusque-là plus facile de trouver dans ces magasins des produits laitiers industriels étrangers que des produits mongols artisanaux (Gardelle \& Ruhlmann 2009). Le délabrement des infrastructures de transformation et de transport consécutif à la fin du régime socialiste en est la principale explication. Alors qu'en 1980 la Mongolie exportait quelque 3800 tonnes de beurre vers l'URSS et ses pays satellites, pour culminer à 4400 tonnes en 1990, elle n'en exporte plus du tout dès le début des années 1990 et doit même en importer pour faire face à la demande de consommation et à la déficience des infrastructures d'acheminement des produits locaux vers les centres urbains.

Pour le lait frais, la production agro-industrielle a elle aussi chuté : elle est passée de 48 millions de litres de lait en 1990 à 25 millions deux ans plus tard, pour s'effondrer à 1,5 million en 1995. Les importations de lait ont doublé entre 2000 et 2004, tandis que le volume des autres produits laitiers importés a été multiplié par cinq (Rossabi 2000). Face à cette situation, le gouvernement mongol semble s'être ressaisi, en instaurant par exemple en 1999 une " Révolution blanche » (Cagaan huvisgal) qui porte ses fruits depuis quelques années. Car, en effet, sur les marchés de la capitale, les Mongols peuvent désormais s'approvisionner à volonté en produits laitiers traditionnels et choisir les produits en fonction de leur provenance, certaines régions étant réputées pour leur beurre de yak ou leurs fromages séchés sucrés, etc. Différents autres programmes, qui se sont succédé, visent à encourager la production laitière et carnée conforme au standard international, pour l'exportation, et à valoriser les produits locaux ${ }^{21}$. 
Photo 13. Produits laitiers traditionnels vendus sur les marchés

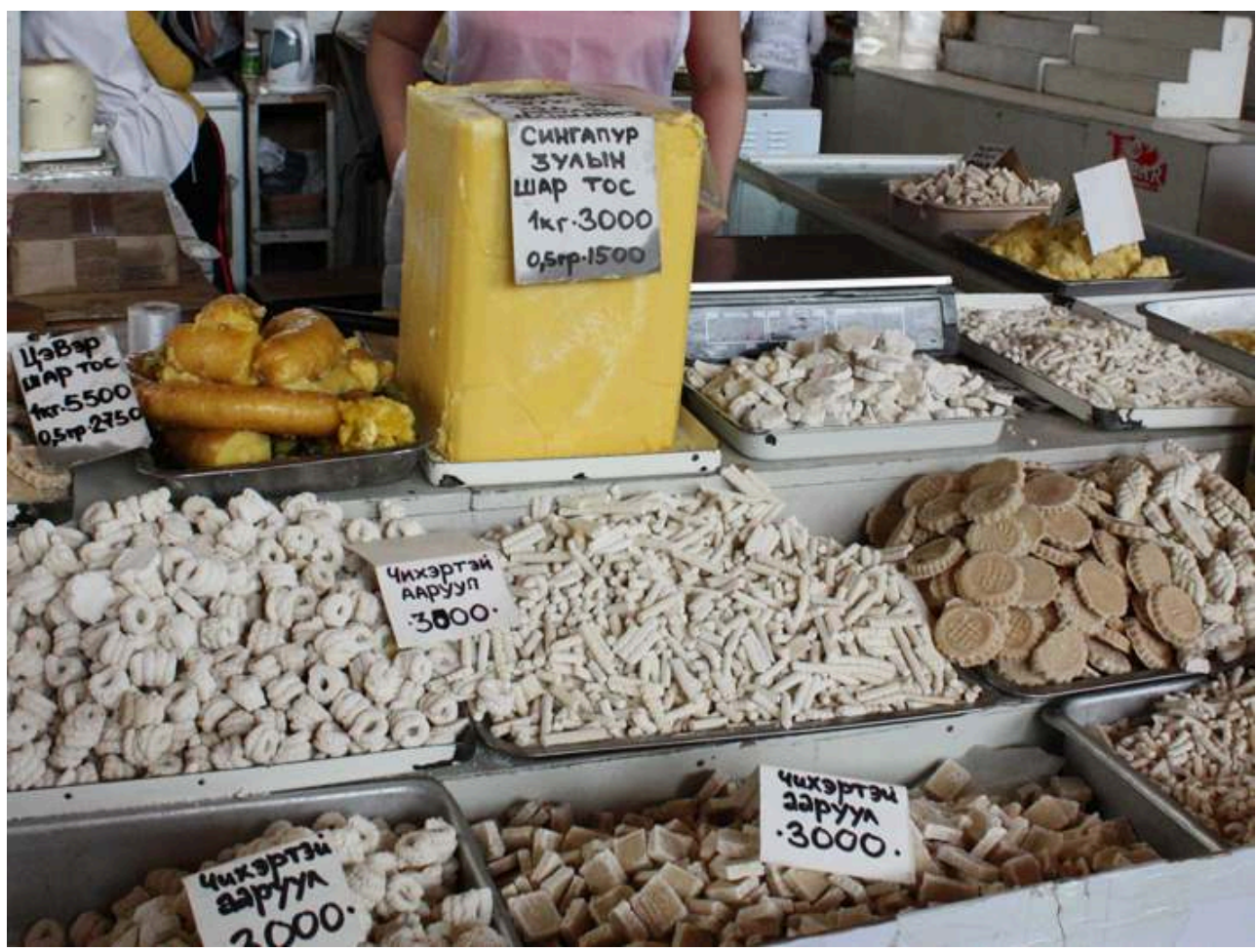

Linda Gardelle (2006)

51 Aujourd'hui, sur les étals des marchés urbains et dans les petits magasins et supérettes de la capitale, les produits laitiers mongols de production industrielle ont repris toute leur place et les importations ont fortement diminué. L'investissement du secteur par des firmes privées et le soutien politique de l'État ont fait que 7 millions de litres de lait ont été produits de manière industrielle en 2008 et que ce chiffre ne cesse d'augmenter ${ }^{22}$.

La route du Millénaire, qui traversera la Mongolie d'Est en Ouest d'ici à quelques années, est aussi un chantier important dans ce domaine, censé participer à l'élaboration d'un milieu économique favorable à la création d'entreprises de divers types en milieu rural. C'est aussi dans ce but qu'ont été mis en œuvre la politique de développement des entreprises (1998) et le programme national de soutien aux petites et moyennes entreprises (1999). Cette politique de développement des entreprises concerne, entre autres, les entreprises d'élevage, et est supposée les encourager à passer d'une transformation primaire à une transformation secondaire par la fabrication de produits finis prêts à la consommation. Le programme national de soutien aux petites et moyennes entreprises, quant à lui, prescrit notamment d'encourager le développement industriel de denrées mongoles susceptibles de se substituer aux importations.

L'organisation des éleveurs en coopératives ou en associations est considérée par les décideurs politiques comme une réponse au manque d'infrastructures et à la désorganisation du transport. La première loi concernant les coopératives a été votée en 1995, créant un environnement législatif favorable à leur apparition. Depuis la loi sur les coopératives (Horšoony tuhaj Mongol ulsyn huul') du 8 janvier 1998, qui se veut un outil pour remédier aux conséquences de l'individualisme des éleveurs, les activités de 
mise en place de ces institutions se sont développées. Ces actions étatiques dans le domaine commercial impliquant la fabrication des produits, leur transport, leur mise sur le marché sont essentiellement de l'ordre de l'encouragement et de l'incitation : les éleveurs sont amenés à prendre en main leur organisation dans un contexte difficile, faiblement sécurisé, économiquement fluctuant, avec un climat aléatoire.

\section{Une industrie laitière innovante en matière de pratiques sociales et culturelles}

L'aube $d u x x^{e}$ siècle laisse entrevoir des évolutions dues au développement de l'industrie laitière mongole. De nouveaux produits laitiers, pasteurisés, conditionnés dans des emballages Tetra Pack "purs » et "sains », ouvriront peut-être la voie à de nouveaux modes de consommation des produits laitiers, à de nouvelles sociabilités. L'entreprise principale Süü XK, anciennement d'État mais aujourd'hui privée, développe l'industrialisation du lait de vache mongole, en axant sa commercialisation sur la diversité des produits en fonction des besoins biologiques des consommateurs et sur la consommation, innovante en Mongolie, du lait, sous-entendu frais et non transformé, bien que pasteurisé ${ }^{23}$. Un film publicitaire montre les membres d'une famille mongole dans un appartement lumineux en train de boire de grands verres de lait à toute heure de la journée et se sentant bien à l'intérieur comme à l'extérieur de leur corps. Beaucoup de femmes pensent qu'en mangeant et en s'appliquant sur le corps et le visage des "aliments blancs", elles auront une belle peau blanche et que cette blancheur extérieure révélera ou favorisera la blancheur, c'est-à-dire la bonté, de leur âme. La terminologie employée par l'entreprise n'est pas innocente et renvoie les Mongols à des notions liées à des pratiques et à des représentations d'ordre rituel et religieux : le lait est qualifié de "pur ». L'entreprise décline tous les laits possibles : un lait nouveau biologique, un lait nourrissant (enrichi en œufs et céréales), un lait au cynorrhodon recommandé aux femmes (pour l'élasticité de leur peau et un affinement de leur silhouette), un lait enrichi en calcium recommandé aux enfants (pour leur croissance, pour rendre leurs articulations flexibles et leurs os forts, ceci renvoyant directement aux rites de naissance, le bouillon de toilette du nouveau-né ayant la propriété de lier ses os et de les fortifier). L'entreprise produit également du beurre de baratte longue conservation, de la crème fraîche épaisse qui guérit de l'anémie ou des problèmes digestifs et stimule l'appétit, ainsi que des yaourts: yaourts onctueux pasteurisés, "yaourt nouveau » aromatisé, yaourt enrichi en probiotiques et yaourt crémeux pour soigner les problèmes d'hypertension artérielle, l'ostéoporose, la sclérose en plaques, etc. Nous constatons que, comme dans les pays occidentaux et plus récemment en Chine, l'accent est mis sur la santé. Le lait est directement corrélé à des questions d'esthétique et de santé. Le slogan «Got milk» diffusé par l'entreprise Süü XK - c'est en fait un slogan américain créé en 1993 par une agence de publicité californienne - incite les Mongols à consommer des produits laitiers mongols industriels sans pour autant rompre avec des traditions.

Il sera intéressant de voir si ces évolutions parviendront à modifier, et dans quelle mesure, les pratiques alimentaires des Mongols urbains et non urbains, sédentaires et nomades, et si des familles les intégreront, et de quelles manières, dans des pratiques rituelles ou ritualisées où les "aliments blancs » traditionnels et artisanaux jouent un rôle structurant et ancrent efficacement des croyances dans le quotidien. 


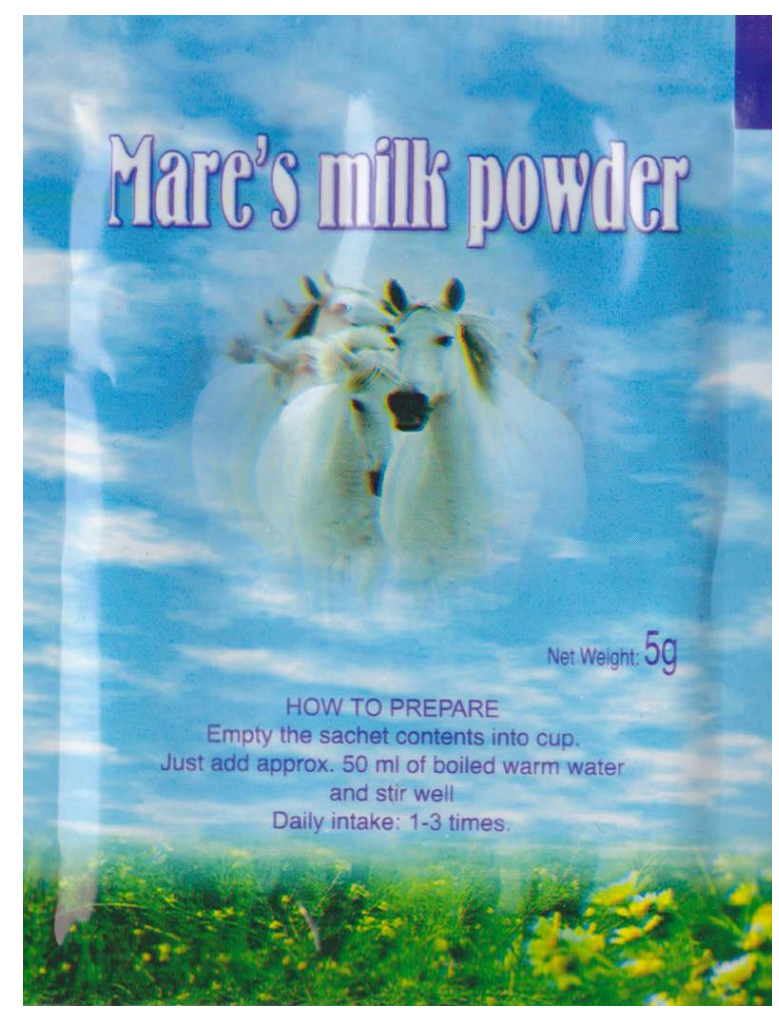

Recto en anglais, verso en mongol

Sandrine Ruhlmann

Les techniques mongoles de traitement du lait et de fabrication des produits laitiers constituent un mode de gestion d'une matière vivante, le lait. Elles sous-tendent des pratiques de commensalité du lait et de ses dérivés. Ces pratiques d'offrande et de consommation des laitages s'insèrent dans un système plus vaste de pratiques sociales et identitaires qui sans cesse sont adaptées au contexte historique. L'étude de la commensalité reliée au lait permet d'appréhender les usages sociologiques des produits laitiers. C'est également un moyen de comprendre comment les pratiques peuvent évoluer, comment le lait sous sa forme liquide non transformée, c'est-à-dire à l'état cru aux yeux des Mongols, pourra prendre place et faire sens dans un système alimentaire qui jusqu'ici ne concevait pas la consommation du lait autrement que sous la forme de laitages: comment le lait, certes pasteurisé, mais bu froid dans un grand verre parviendra à remplacer la traditionnelle boisson, le thé au lait ? Les Mongols feront-ils un jour des libations de lait pasteurisé?

Il sera intéressant de voir également si, dans quelques années, les Mongols utiliseront le lait et ses dérivés gras dans leur cuisine - du beurre comme élément de cuisson, de la crème pour composer une sauce -, et pas seulement comme aliment à ajouter dans le thé ou les bouillies de céréales d'origine tibétaine, et quelle place ils accorderont à cette nouvelle cuisine. Car les Mongols, en bons éleveurs nomades, possèdent un savoir-faire de transformation du lait en produits laitiers, mais ils n'ont pas pour habitude d'utiliser du lait, ni même de la crème ou du yaourt, dans des préparations culinaires autres que la confection de bouillies. 


\section{BIBLIOGRAPHIE}

Accolas, J-P., Deffontaines, J-P. \& Aubin, Fr.

1975 Les activités rurales en République populaire de Mongolie. I. Agriculture et élevage.

II. Produits laitiers, Études mongoles 6, pp. 7-98.

Accolas, J-P. \& Deffontaines, J-P.

1976 Quelques données sur l'élevage du yak en République Populaire de Mongolie, Ethnozootechnie 15, pp. 133-141.

1978 Le lait et les produits laitiers en République populaire de Mongolie, Le Lait, Revue générale des questions laitières LVIII(575-576), pp. 276-286.

Bianquis-Gasser, I.

1996 Pratique autour des laitages dans la sociabilité mongole contemporaine, in Comment peut-on être socio-anthropologue. Autour d'Henri Hatzfeld (L'Harmattan), pp. 149-166.

Gardelle, L.

2010 Mongolian Politicians and their Herds. The Influence of the Elite of Rural Origins,

Monumenta Serica Monographs Series LXI, Miscellanea Asiatica, Mélanges en l'honneur de Françoise Aubin, sous la direction de D. Aigle, I. Charleux, V. Goossaert et R. Hamayon, pp. 173-184.

Gardelle, L. \& Ruhlmann, S.

2009 La revalorisation de l'élevage nomade en Mongolie. Des logiques économiques, écologiques et culturelles, Autrepart 50, Des produits de terroir pour conserver la diversité biologique et culturelle au Sud ? Enjeux, acteurs, instruments, M-Ch. Cormier-Salem et B. Roussel (eds.), pp. 135-152.

Hamayon, R.

1977-2 Il n'y a pas de fumée sans dieu, L'Ethnographie 74-75, numéro spécial - Voyages chamaniques, pp. 171-188.

1978 Des fards, des mœurs et des couleurs. Étude d'ethno-linguistique mongole, in S. Tornay (éd.), Voir et nommer les couleurs (Nanterre, Laboratoire d'ethnologie et de sociologie comparative), pp. 207-247.

Histoire secrète des Mongols. Chronique mongole du XIII ${ }^{e}$ siècle 1994 Traduit du mongol, présenté et annoté par M-D. Even et R. Pop, préface de R. Hamayon (Gallimard).

Plan Carpin, J. de

1965 Histoire des Mongols. Traduit et annoté par J. Becquet et L. Hambis (Paris, Adrien-

Maisonneuve).

Polo, M.

1988 Le livre des merveilles. Texte intégral en français moderne d'après les travaux de M. G. Pauthier sur les manuscrits de la bibliothèque nationale de Paris avec les 84 enluminures exécutées pour le Duc de Bourgogne en 1399 (Librairie de Jean De Bonnot).

Rintchen, Y.

1977-2 Pourquoi on offre des chevaux et des moutons aux esprits chamaniques Mongols, L'Ethnographie 74-75, numéro spécial - Voyages chamaniques, pp. 155-156.

Rossabi, M.

2000 "Introduction", in Ts. Namkhainjambuu, Bounty from the Sheep : Autobiography of a Herdman (Cambridge, The White Horse Press). 
Rubrouck, G. de

1993 Voyage dans l'empire mongol : 1253-1255. Traduit et commenté par Cl. et R. Kappler (Imprimerie Nationale).

Ruhlmann, S.

2007 Les «nourritures enveloppées » du Mois blanc : nourritures de fête du nouvel an lunaire en Mongolie, OCHA. (en ligne www.lemangeur-ocha.com)

2008 Une curieuse pâtisserie en forme de semelle : le nouveau support de bonheur des Mongols halh bouddhisés, Anthropology of Food, So, Varia, le numéro permanent. (en ligne www.aof.revues.org)

2009a Une soupe peu ordinaire. Analyse du repas des funérailles chez les Mongols, Techniques et culture 51, pp. 247-271.

2009b L'enterrement chez les Mongols contemporains : le cercueil, la tombe et la yourte miniature du mort, EMSCAT 40. (en ligne www.emscat.revues.org)

2010 Les rites de naissance chez les Mongols Halh : la fermeture/ouverture des corps, des nourritures et du social, Monumenta Serica Monographs Series LXI, Miscellanea Asiatica, Mélanges en l'honneur de Françoise Aubin, sous la direction de D. Aigle, I. Charleux, V. Goossaert et R. Hamayon, pp. 225-247.

2013a Le lait, ses dessus et ses dessous. Technologie du lait et de ses dérivés en Mongolie contemporaine, OCHA (à paraître en ligne)

2013b Are buuz and banš traditional Mongolian foods ? Strategy of appropriation and identity adjustment in contemporary Mongolia (Oxford University Press). (à paraître)

\section{NOTES}

1. Un autre article d'Accolas et Deffontaines a été consacré aux techniques laitières (1978) et un autre plus spécifiquement à l'élevage du yak (1976).

2. Nous publierons un article ethnographique sur les technologies laitières artisanales domestiques contemporaines (Ruhlmann 2013a, à paraître).

3. Cette même louche qui sert à aérer le lait pour le faire mousser et fabriquer de la peau crémeuse (öröm).

4. Selon le témoignage de Plan Carpin, la notion de prémices étaient déjà très importante au XIII siècle. Il nous explique que les Mongols offraient à des figurations d'esprits les «premiers laits des troupeaux et des juments » $(1965$, p. 36).

5. Commentaires de Cl. et R. Kappler (1993, pp.168-169, 248, note 1 chapitre 35) au récit de Guillaume de Rubrouck.

6. Le « beurre blanc » se compose de farine de blé grillée sans avoir roussi, à laquelle on mêle du lait chaud et de la peau crémeuse du lait (öröm). Ce mélange est additionné de filaments de fromage séché émiettés (eezgij) et de sucre en poudre.

7. Sur la considération ambivalente des raviolis chez les Mongols, $c f$. Ruhlmann 2007, 2013b.

8. Selon le témoignage de Guillaume de Rubrouck traduit et annoté par Cl. et R. Kappler (1993, pp. 84-85), à la fin de la période estivale de lactation, les femmes constituaient des provisions de fromages et de caillé séchés. Ainsi en hiver, ne disposant pas ou que peu de lait frais, les femmes dissolvaient du caillé dans de l'eau chaude. L'eau devenait acide et les nomades la buvaient en remplacement du lait bouilli ou fermenté.

9. Les Mongols confèrent aujourd'hui aux "gâteaux-semelle » (ul boov) une qualité et une valeur de support de bonheur qu'ils attribuaient exclusivement, à une haute époque et jusqu'à leur conversion au bouddhisme à la fin $\mathrm{du} \mathrm{XVI}^{\mathrm{e}}$ siècle, à des parts de viande rattachées à l'os (Ruhlmann 2008). 
10. Certaines femmes rapportent le cordon ombilical et le rangent dans une pochette en soie au fond d'un coffre au nord de la yourte (Ruhlmann 2010).

11. C'est cette même part de viande que les mariés se partagent le jour de leur mariage pour augurer favorablement de la solidité de leur union.

12. La famille consulte tout de même un moine astrologue pour des précisions d'ordre rituel, comme la couleur du linceul et l'emplacement de la tombe au cimetière (Ruhlmann 2009b).

13. Les Mongols disent qu'il est également offert aux chiens qui sont, dans la religion bouddhique, les gardiens de l'âme, afin que l'âme du mort ne renaisse pas dans un chien ou un autre animal, mais dans le corps d'un humain.

14. Concernant cette soupe funéraire peu ordinaire, $c f$. Ruhlmann 2009a.

15. Il semble que le feu n'intervient aujourd'hui que sous forme de fumée, dans les rites de purification dans les cas de maladie, de mort et d'accouchement difficile : le clergé bouddhique et les chamanes purifient par fumigation, respectivement avec de la poudre et des branches de genévrier ou d'épicéa (Hamayon 1977-2).

16. Au XIII ${ }^{\mathrm{e}}$ siècle, Guillaume de Rubrouck a pu observer que seuls les hommes étaient chargés de traire les juments et de baratter le lait; le barattage s'effectuait avec un "bâton gros à sa base comme la tête d'un homme » et « creux par-dessous » (1993, pp. 84-85).

Chaque jour, le lait cru des traites est ajouté au lait baratté la veille, dans une outre en peau de bœuf (höhü̈r) ou dans un baril d'une contenance de 50 ou 100 litres. La fabrication est continue et l'addition quotidienne des traites compense la consommation journalière. Le récipient n'étant pas lavé, il comporte sur ses parois internes des bactéries, levures et moisissures favorisant le processus semi-continu de fermentation acido-alcoolique du lait à température ambiante. La batte à beurre ou un large bâton sont utilisés comme agitateur. Les éleveurs disent qu'il faut mille coups de batteurs pour obtenir un "bon ajrag", c'est-à-dire une bonne fermentation lactique.

17. Toutes sortes d'objets étaient placés dans les tombes des hommes de rang supérieur pour qu'ils vivent dans l'au-delà une vie similaire à leur vie sur terre : une demeure, une jument pour avoir du lait et faire de l'élevage, un cheval pour le monter, de la viande pour se nourrir (Plan Carpin 1965, p. 41 et note 28 p. 146).

18. Plan Carpin explique qu'à cette haute époque, les Mongols se nourrissent l'été de lait de jument fermenté et de viande de gibier, et l'hiver de viande en petite quantité dans du bouillon salé. Seuls les riches consomment et boivent du lait de jument fermenté l'hiver (1965, pp. 48-49). Rubrouck ajoute qu'en été, les Mongols mangent également de la viande séchée au soleil et au vent, ainsi que les intestins bouillis de chevaux (1993, p. 156).

19. Jusqu'au début du $x^{e}$ siècle, pour marquer la clôture de la période de traite des juments à l'automne, ainsi que la fin de la fabrication du lait de jument fermenté, les Mongols célébraient le "relâchement des juments » (Bianquis-Gasser 1996).

20. Fête à l'occasion de laquelle les Mongols assistent et participent à des compétitions locales et/ ou nationales de lutte, de tir à l'arc et de courses de chevaux.

21. On peut citer notamment : le programme national d'amélioration de l'élevage et de la qualité du bétail (1997), censé favoriser l'adaptation de l'élevage à l'économie de marché et améliorer la qualité et la productivité du bétail ; le programme national de la Révolution blanche (1999), qui a pour but l'augmentation de la production industrielle des produits laitiers mongols et l'amélioration de leur place sur le marché; le projet d'exportation de viande (1999), qui veut augmenter la production des produits carnés et leur exportation en améliorant leur qualité afin de les conformer aux standards internationaux; le programme d'encouragement aux industries d'exportation (1999), qui lui aussi entend permettre l'adaptation des marchandises aux marchés mondiaux; le programme cachemire (2000), ayant pour but la hausse de l'exportation de cachemire non brut; le programme laine (2001) voulant moderniser la production et augmenter la capacité des usines ; et le programme cuir (2001), destiné à améliorer la qualité des produits 
pour favoriser leur exportation. Tous ces programmes sont référencés, en mongol, sur le site du ministère de l'Agriculture de Mongolie (www.pmi.gov.mn).

22. Chiffres du Mongol Ulsyn Statistikijn Emhetgel 2008.

23. http://www.mongolmilk.mn

\section{RÉSUMÉS}

Les éleveurs nomades mongols tirent leur subsistance de l'élevage extensif de plusieurs espèces animales. Cet article est consacré à l'étude des modalités de consommation des produits laitiers. Il présente les usages rituels ou ritualisés de ces produits dans des contextes ordinaires et de célébration d'événements de la vie sociale de familles d'éleveurs. Nous mettrons en évidence comment les Mongols confèrent aux dérivés du lait une valeur sociale et un fort contenu symbolique. Depuis deux décennies, la politique de l'État postcommuniste encourage les éleveurs, en érigeant au rang d'emblème national les produits issus de l'élevage, activité qui reste la principale richesse du pays. À travers les pratiques de consommation et d'usage des produits laitiers, tout un système métaphysique proprement mongol ainsi qu'un système de relation entre les vivants d'une part, entre les vivants, les morts et les entités naturelles et surnaturelles d'autre part, se révèlent.

Mongolian nomads derive their livelihood from an extensive breeding focused on several animal species. This article is devoted to the study of the consuming modalities of dairy products; it presents the ritual or ritualized uses of these products in the contexts of ordinary events and celebration of social events among herder families. We will highlight the way the Mongols give a sociological value and a high symbolic content to milky derivatives. Since two decades, a PostCommunist State policy encourages herders by rising at the rank of national emblem products from breeding, this activity being the main wealth of the country. A properly Mongolian metaphysical system and a system of relation between humans, between the living and the dead and the natural and supernatural entities, reveal themselves through consumption and use practices of dairy products.

\section{INDEX}

Mots-clés : alimentation, consommation, emblème, hospitalité, lait, laitages, politique, postcommuniste, rituel

Index géographique : Mongolie

Keywords : alimentation, animal, breeding, Buddhism, consumption, dairy products, emblem, hospitality, milk, nationalism, politics, Post-Communist, ritual, Shamanism

nomsmotscles Halh

Thèmes : animal, bouddhisme, chamanisme, élevage, nationalisme 


\section{AUTEURS}

\section{SANDRINE RUHLMANN}

Docteur en anthropologie sociale et ethnologie. L'auteur a soutenu en 2006 à l'EHESS une thèse sur les pratiques alimentaires chez les Mongols Halh. Elle poursuit actuellement des recherches sur les techniques et les savoirs mongols dans le domaine de l'alimentation, en se concentrant sur les changements qui s'opèrent dans la capitale, Ulaanbaatar. Post-doctorante au Laboratoire d'anthropologie sociale (Collège de France, Paris), elle étudie actuellement la perception des maladies animales chez les éleveurs. À travers l'observation et l'analyse des pratiques alimentaires, différentes conceptions relatives à la culture mongole sont étudiées, comme celles de la mort, de la naissance, de l'âme, ou encore de l'espace domestique, de l'hospitalité et du partage.

sandrine.ruhlmann@college-de-france.fr

\section{LINDA GARDELLE}

Sociologue, docteur de l'Université Paris 1 Panthéon-Sorbonne. Sa thèse, soutenue en 2007, était consacrée aux mutations vécues par le pastoralisme nomade en Mongolie depuis la fin du régime socialiste. Aujourd'hui enseignant-chercheur dans une école d'ingénieurs, l'ENSTA-Bretagne, elle poursuit ses recherches sur les questions de développement, d'identité et de gouvernance au sein du Centre de Recherche sur la Formation (EA 1410). Elle a notamment publié Pasteurs nomades de Mongolie (2010, Buchet-Chastel).

linda.gardelle@ensta-bretagne.fr 